\title{
JUPITER'S RADIATION BELTS AND THE SWEEPING EFFECT OF ITS SATELLITES
}

\section{GILBERT D. MEAD WILMOI N. HESS}

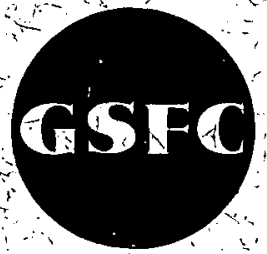

\section{AUGUST 1972}

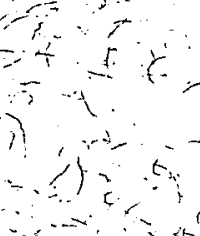

\section{GODDARD SPACE FLIGHT TR ER}

\section{GREENBELT, MÁY YAND}
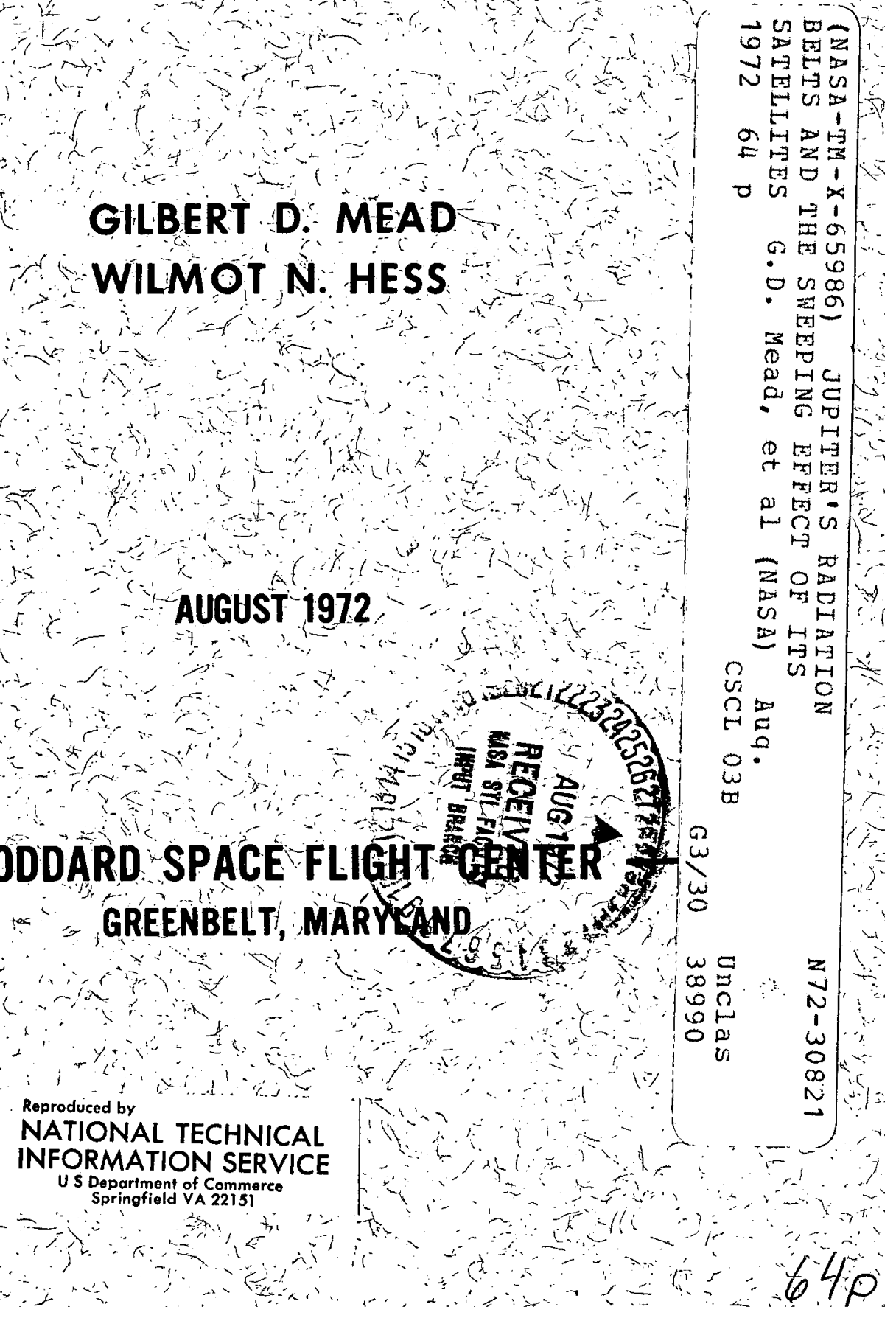


\begin{abstract}
JUPITER'S RADIATION BELTS AND THE
SWEEPING EFFECT OF ITS SATELLITES
\end{abstract}

\author{
Gilbert D. Mead \\ Laboratory for Space Physics \\ NASA Goddard Space Flight Center \\ Greenbelt, Maryland 20771 \\ Wilmot N. Hess \\ Environmental Research Laboratories \\ National Oceanic and Atmospheric Administration \\ Boulder, Colorado 80302
}

August 1972

Submitted to the Journal of Geophysical Research $i$ 


\begin{abstract}
Jupiter's electron and proton radiation belts are analyzed, with particular reference to the sweeping effect of its five inner satellites, located deep within its magnetosphere. The characteristics of trapped electrons and protons with a magnetic moment of $50 \mathrm{Mev/gauss,} \mathrm{considered} \mathrm{typical} \mathrm{at} \mathrm{Jupiter,} \mathrm{are} \mathrm{calculated.}$ The assumption is then made that a particle would be removed from the radiation belt if, in its normal motion, it would happen to impact a satellite. The mean absorption time before impact is calculated for particles located at the radial distance of each of the satellites. This average lifetime was found to be of the order of a few days. A characteristic diffusion time near each satellite was calculated, assuming violation of the third invariant due to magnetic fluctuations associated with fluctuations in the solar wind. This diffusion time was found to be long compared with the absorption lifetimes at Europa $(L=9.5)$, Io $(L=6.0)$, and Amalthea $(\mathrm{L}=2.5)$. Assuming that this process is dominant, the se three satellites are expected to act as a barrier to the inward diffusion of solar wind particles, and fluxes of trapped protons would be reduced by two or three orders of magnitude just inside the orbit of Europa; very few could diffuse past Io or Amalthea. Due to the ten-degree tilt of the magnetic dipole with respect to the rotational axis, however, particles with equatorial pitch angles greater than $69^{\circ}$ would have significantly longer absorption lifetimes, and a much larger fraction could escape absorption by a satellite. We believe that this is the most likely explanation for the observed highly-peaked pitch-angle distributions of trapped electrons in the region of the decimeter emission, just inside the orbit of Amalthea. A flyby mission for which the lowest possible radiation levels are desired should be planned so that the spacecraft remains at relatively high magnetic latitudes near perijove.
\end{abstract}


PRECEDING PAGE BLANK NOT FILMED

CONTENTS

Page

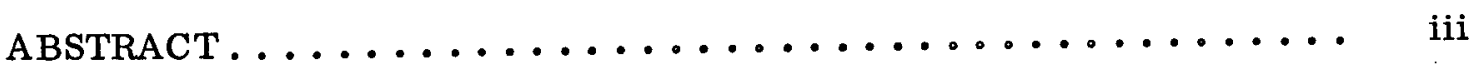

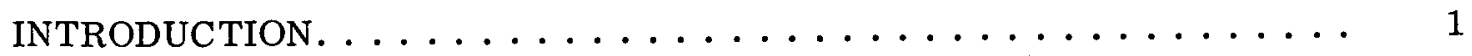

JUPITER'S MAGNETIC FIELD AND MAGNETOSPHERE . . . . . . 9

CHARACTERISTICS OF JUPITER'S INNER SATELLITES . . . . . . 11

TRAPPED-PARTICLE MOTION AT JUPITER ............. 15

SWEEPING EFFECT OF JUPITER'S SATELLITES. . . . . . . . 21

RADIAL DIFFUSION RATES AT JUPITER ............ 28

EFFECT OF THE DIPOLE TILT. ............... 39

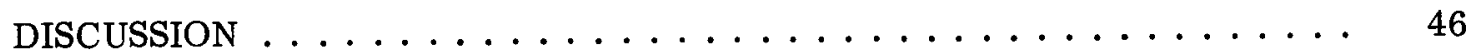

SUMMARY AND CONCLUSIONS . . . . . . . . . . . . . . 49

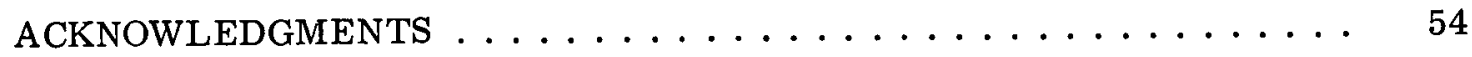

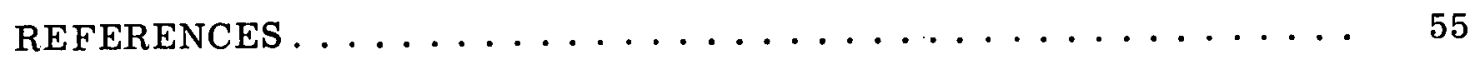




\section{ILLUSTRATIONS}

$\underline{\text { Figure }}$

The $10.4-\mathrm{cm}$ radio brightness distribution measured by Berge (1966) with a two-antenna interferometer. The peaks of the distribution appear to be about $0.9 \mathrm{R}$, from the edge of the disc. The bars in the lower right indicate the resolution of the instrument .............

The position of Jupiter's five inner satellites in its magnetosphere. Diameters are not to scale .........

3 Co-rotating jovimagnetic coordinate system. Jupiter and the thermal plasma are stationary in this system. The inner satellites appear to revolve in a retrograde direction with an inclination of $10^{\circ}$ and periods of about 10-13 hours (60 hours for Amalthea).................. 20

4 Sketch used to calculate absorption and diffusion times near a satellite with diameter $\mathrm{D}_{\mathrm{s}} \ldots \ldots \ldots \ldots . \ldots . \ldots 23$

$5 \quad$ Characteristic absorption and diffusion times near each satellite. Diffusion dominates near Callisto, but absorption dominates at Amalthea, Io, and Europa. . . . . . . . . . 26

6 Sketch of expected off-equatorial proton fluxes at Jupiter, assuming diffusion is caused by magnetic fluctuations. CRAND is ignored. Fluxes of inward-diffusing protons are expected to be reduced by several orders of magnitude at Europa ( $L=9.5)$, and none should diffuse past Io or Amalthea. . 36

$7 \quad$ The fraction $F$ of particles that can diffuse in past Europa without being absorbed, plotted as a function of pitch angle. The characteristic diffusion lifetime is assumed to be 24 days. The increase at $69^{\circ}<a_{0}<111^{\circ}$ is due to the fact that during a substantial fraction of these particles' drift period, the satellites appear to pass by at latitudes above or below their mirror points ................ 44 


\section{INTRODUCTION}

The planet Jupiter is known to have a strong magnetic field and an intense belt of trapped electrons. It probably has a proton radiation belt as well, although it is not possible to estimate the proton intensities from Earth-based measurements. The purpose of this paper is to determine some of the probable characteristics of Jupiter's electron and proton radiation belts, using observations where available to determine known quantities, and applying theoretical concepts based on our understanding of the Earth's radiation belts to predict characteristics which have not yet been measured directly. We give particular consideration to the possible effects of Jupiter's five inner satellites, located deep within Jupiter's magnetosphere. These satellites exert a sweeping effect, removing trapped particles which impact them. Thus it is likely that actual particle fluxes in some regions of Jupiter's magnetosphere are much lower than they would be in the absence of any satellites. We begin by reviewing our present knowledge of Jupiter's radiation belts.

Trapped electrons. Nonthermal emission in the decimeter wavelength region was first discovered by Sloanaker (1959) at $3000 \mathrm{MHz}(10 \mathrm{~cm})$. More

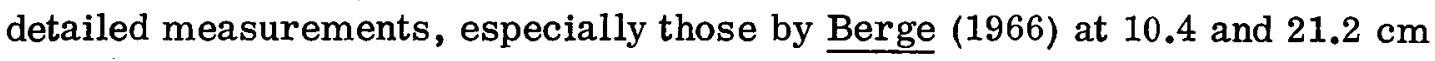
and by Bransom $(1968)$ at 75 and $21 \mathrm{~cm}$, clearly established that this decimetric radiation is primarily emitted not from the surface of the planet itself, but from a region near the equatorial plane, somewhat removed from the surface. The 10.4-cm brightness distribution as measured by Berge (1966) is shown in Figure 1. Distinguishing characteristics of this radiation component are its nonthermal spectrum, the relatively large extent and distinctive shape of the 


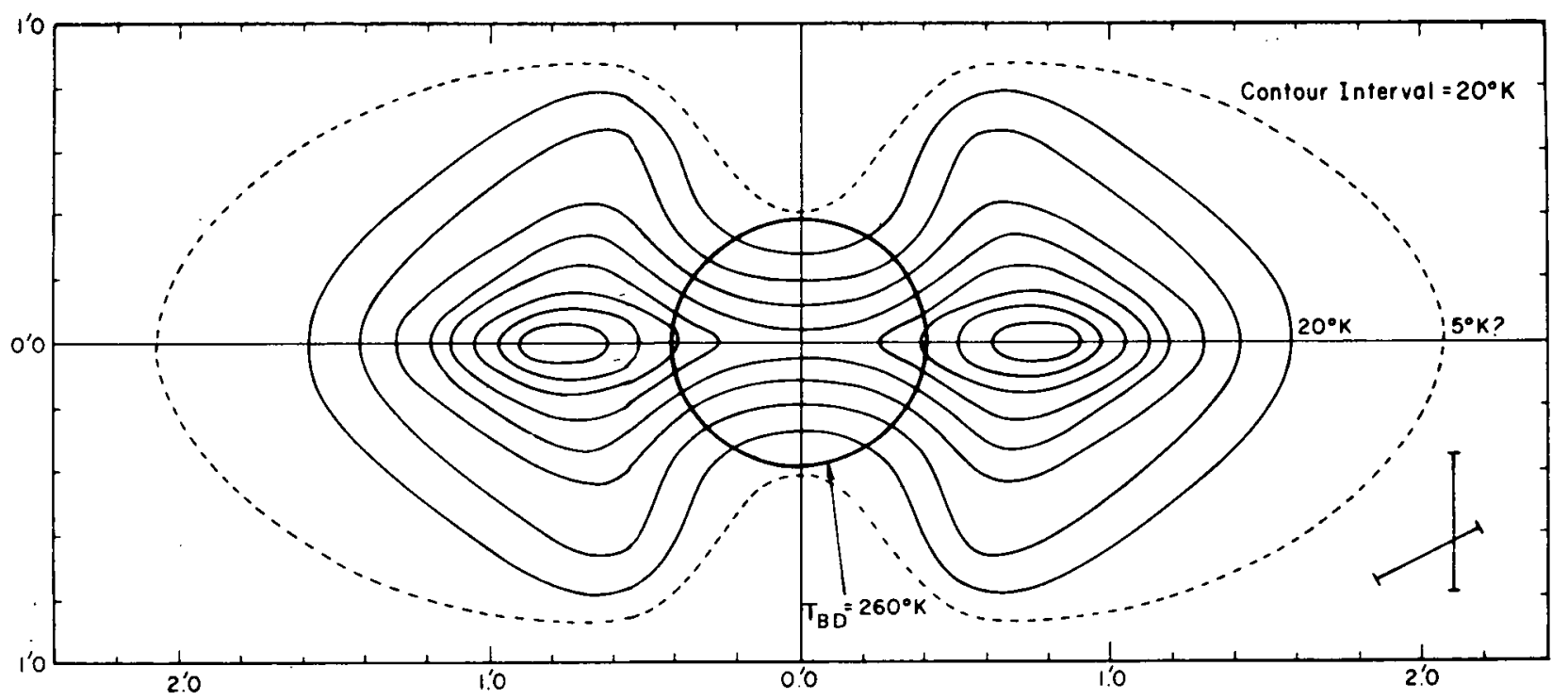

Figure 1. The 10.4-cm radio brightness distribution measured by Berge (1966) with a two-antenna interferometer. The peaks of the distribution appear to be about $0.9 \mathrm{R}$, from the edge of the disc. The bars in the lower right indicate the resolution of the instrument. 
emitting region, the relatively high degree of linear polarization (approximately $22 \%$ ) but almost complete absence of circular polarization, and the fact that it is to some degree beamed in a preferred direction. Extensive reviews of the radio emission from Jupiter at both decimeter and decameter wavelengths have been published by Roberts and Komesaroff (1965), Warwick $(1964,1967,1970)$, and Carr and Gulkis (1969).

Early analyses of this decimetric radiation by Field (1959), Chang and Davis (1962), Thorne (1965), and others established quite clearly that it is synchrotron radiation from trapped electrons spiraling around the Jovian magnetic field lines. Chang and Davis (1962) were able to explain the radiation as being emitted from electrons with energies in the 5-75 Mev range and densities of the order of $10^{-3}$ to $10^{-2} \mathrm{~cm}^{-3}$, with fields of $0.1-1$ gauss in the emitting region. In a more recent analysis, Warwick (1970) finds that the observed decimeter radiation could be produced by 6-Mev electrons with a peak density at $1.8 \mathrm{R}_{\mathrm{J}}$ near the equator of $1.6 \times 10^{-4} \mathrm{~cm}^{-3}$ and omnidirectional flux of $4.8 \times 10^{6} \mathrm{~cm}^{-2} \mathrm{sec}^{-1}$, radiating in a field of about 2 gauss. The average electron radiation lifetime would be about 0.3 years. Luthey and Beard (1972), by comparing the radio emission at 10.4 and $21-\mathrm{cm}$ wavelengths, concluded that the emission could be produced by equatorial electrons with energies of 5-70 Mev and densities of about $1.5 \times 10^{-3}$ $\mathrm{L}^{-5.4} \mathrm{~cm}^{-3}$, where $\mathrm{L}$ is the radial distance in units of Jovian radii. At the Jupiter Radiation Belt Workshop held at the Jet Propulsion Laboratory in July, 1971, a workshop model of relativistic electrons was determined (Divine, 1971, 1972; Beck, 1972). This model shows a nominal electron belt with a flux of $2 \times 10^{7} \mathrm{~cm}^{-2} \mathrm{sec}^{-1}$ out to $\mathrm{L}=2$ and a gradual falloff outside $\mathrm{L}=2$. An upper limit model was a factor of 3 higher in flux. 
The equatorial pitch-angle distribution of the electrons in the emission region must be sharply peaked near $\alpha_{e}=90^{\circ}$, as first pointed out by Chang and Davis (1962). Two observational facts lead to this conclusion: (1) the $22 \%$ linear polarization of the decimeter emission, with the maximum of the E-vector lying in the magnetic equatorial plane, rather than perpendicular to it; and (2) the beaming of the emission in the magnetic equatorial plane. The total beamed intensity is observed to drop approximately $11 \%$ as observed from a magnetic latitude of $\pm 13^{\circ}$, as compared with the equatorial intensities (Roberts and Komesaroff, 1965). Electrons with relatively flat helices are required to fit these observations.

Thorne (1965) analyzed the Roberts and Komesaroff data and concluded that a two-component model of the trapped electrons was required to simultaneously fit the observed polarization and beaming at $21 \mathrm{~cm}$. One component is relatively isotropic, and the other has extremely flat pitch angles. If one assumes an equatorial pitch-angle distribution proportional to $\sin \mathrm{q}_{\alpha_{e}}$, Thorne concluded that the first component must have $\mathrm{q} \lesssim 2$ and the second must have $\mathrm{q} \gtrsim 20$. He found that a distribution proportional to $\sin ^{2} \alpha_{e}+2.0 \sin ^{40} \alpha_{e}$ was capable of fitting the 21-cm data. Similar conclusions were reached in the recent study by Clarke (1970).

No completely satisfactory explanation has been offered for the presence of electrons with such sharply-peaked equatorial pitch angles. Chang and Davis (1962) pointed out that inward diffusion of particles by violation of the third invariant, while the first and second invariants are conserved, would leak to a preponderance of electrons with flat helices. Nakada et al. (1965) calculated this effect, but their results do not indicate nearly so much peaking as the observations seem to require. In this study we shall suggest an alternate explanation 
in terms of electrons with flat pitch angles which are much more capable of diffusing in past the inner satellites without being absorbed, due to the $10^{\circ}$ tilt of the magnetic dipole with respect to the rotational axis.

Chang and Davis (1962) estimated that the time scale for diffusing solar-wind electrons into the region of radio emission by violation of the third invariant was of the order of a million years, assuming that the diffusion was caused by variations in the solar wind strength and changes in the magnetopause position. However, the electron lifetime at $\mathrm{L}=2$ due to synchrotron loss is only about a year. Some additional process, therefore, seems to be needed for the electrons besides radial diffusion from magnetopause motion, but the nature of this process is presently not understood. It may be related to the storm-time processes that rapidly energize and move electrons in the Earth's radiation belt. Electrons trapped in the Earth's magnetosphere display frequent non-adiabatic time variations (ess, 1968). They exhibit large temporal fluctuations, short lifetimes, large changes due to magnetic storms, and pitch-angle scattering. In his survey paper on magnetospherically-trapped particles, Williams (1972) stressed the difficulty in obtaining meaningful time averages of electron fluxes, because of the great fluctuations in electron intensities observed throughout the trapping region. In one well-documented case (Williams et al., 1968), relativistic electrons appeared to have been injected at $L=3.0-3.5$, deep within the trapping region, during a major magnetic storm.

Protons in the Earth's radiation belt do not exhibit such rapid temporal fluctuations. In general, the electrons show a much more complicated behavior than do the protons, and several additional processes act on the electrons that 
do not operate on the protons. We have little quantitative understanding of these additional processes. It would not be surprising, then, to find that Jupiter's electrons also behave in unpredictable ways.

Trapped protons. Nothing is known experimentally about trapped protons at Jupiter, because protons radiate synchrotron emission far less efficiently than do electrons; they are therefore undetectable by Earth-based measurements. We shall, however, review our understanding of the energetic protons in the Earth's radiation belt and use this to study Jupiter.

The terrestrial outer-zone protons first measured by Davis and Williamson (1963) from $L=2$ to $L=7$, with energies between $100 \mathrm{kev}$ and $5 \mathrm{Mev}$, have a very orderly nature and are now quite well understood (Hess, 1968). Nakada et al. (1965) showed that these protons have diffused radially inwards from the magnetopause, conserving the first two adiabatic invariants $\mu$ and J. These protons have an exponential spectrum

$$
\mathrm{j}(>\mathrm{E})=\mathrm{ke}^{-\mathrm{E} / \mathrm{E}_{0}}
$$

and the characteristic energy of the particles shows a steady, consistent behavior

$$
\mathrm{E}_{0}=\mathrm{cL}^{-3}
$$

for $90^{\circ}$ pitch-angle particles at $\mathrm{L}<6$. For other equatorial pitch angles, $\mathrm{E}_{0}$ is a different but predictable function of L (Nakada et al., 1965). The agreement between theory and observation shows that radial diffusion without pitch angle scattering is the dominant dynamic process for these protons. Nakada and Mead (1965) and Tverskoy (1965) calculated equilibrium fluxes of outer-belt protons, 
assuming a source near the magnetopause, radial diffusion, and atmospheric loss. The predicted fluxes agreed rather well with the measurements. Williams (1972) concluded that the behavior of both the steady-state and non-steady state outerbelt protons could be satisfactorily described by radial diffusion, with a value of the diffusion coefficient $D$ equal to $2.4 \times 10^{-9} L^{10} R_{E}^{2} /$ day.

The energetic protons in the Earth's inner radiation belt are now also fairly well understood. A major source of these protons has long been considered to be cosmic ray albedo neutron decay (CRAND). In a recent study Farley and Walt (1971) analyzed source and loss processes of inner-belt protons at $\mathrm{L}<1.7$. Using CRAND, energy-loss collisions, and radial diffusion by violation of the third invariant, they calculated proton flux intensities, energy spectra, and radial distributions. They found good agreement with observations for equatoriallytrapped protons with first invariant $\mu$ between 200 and $3000 \mathrm{Mev} /$ gauss. The radial diffusion coefficient required $\left(1.0 \times 10^{-8} \mathrm{~L}^{10} \mathrm{R}_{\mathrm{E}}^{2} /\right.$ day or $6.4 \times 10^{-6} \mathrm{~L}^{10} \mu^{-1}$ $\mathrm{R}_{\mathrm{E}}^{2}$ /day) was consistent with those derived by a variety of other methods.

Thus we seem now to have a fairly good quantitative understanding of trapped protons in the Earth's radiation belt. They behave reasonably predictably using straightforward concepts of adiabatic theory. The two major sources seem to be protons in the plasma sheet whose uitimate source is the solar wind (Williams, 1972) and CRAND, with radial diffusion by violation of the third invariant being the main mechanism for adjusting their spatial position.

In this paper we shall assume that protons on Jupiter also behave in predictable ways. The known characteristic of Jupiter's magnetosphere will be 
examined and some of the characteristics of trapped particles which we might expect to find there will be calculated. In particular, the possible effects of Jupiter's satellites on its radiation belts will be studied. Our calculations will be applied to electrons as well as to protons, although we recognize that a number of unknown processes might be operating on electrons. 


\section{JUPITER'S MAGNETIC FIELD AND MAGNETOSPHERE}

The strength of Jupiter's magnetic dipole moment is approximately $4 \times 10^{30}$ gauss $-\mathrm{cm}^{3}$, or 10 gauss $-\mathrm{R}_{\mathrm{J}}^{3}$ (Warwick, 1970). Although several independent arguments lead to a number of this magnitude, perhaps the most convincing one relates to the explanation of the extremely sharp upper-frequency cutoff of the Io-associated decametric emission at $39.5 \mathrm{MHz}$. If we assume that this cutoff frequency is equal to the electron gyrofrequency at the base of a dipole field line passing through Io $(L=5.95)$, we get a surface field of 14.1 gauss at a latitude of about $65^{\circ}$, or somewhat less than 10 gauss at the equator. For the calculations in this paper,we shall assume a field strength in the magnetic equatorial plane equal to $10 / \mathrm{L}^{3}$ gauss. At corresponding $\mathrm{L}-\mathrm{values}$, this is about 30 times greater than the Earth's field.

The Jovian dipole is tilted by about $10^{\circ}$ with respect to the rotational axis. Measurements of the decimetric radiation by Roberts and Komesaroff (1965) show that the plane of polarization rocks back and forth $\pm 10^{\circ}$ relative to the rotational equator as the planet rotates. This indicates that the magnetic equator is inclined by about $10^{\circ}$ with respect to the rotational equator, and the magnetosphere and its trapped radiation exhibit a diurnal wobble similar to the Earth's. Careful measurements by Berge (1972) of the position of the decimetric radiation with respect to the visible planet indicate that the center of the dipole is offset, vertically and horizontally, by less than $0.1 \mathrm{R}_{\mathrm{J}}$.

The high magnetic field strength at Jupiter leads to an extremely large magnetosphere. From considerations of pressure balance, the distance to the 
magnetopause is proportional to $\left(\mathrm{B}^{2} / \mathrm{m} \mathrm{n} \mathrm{v}^{2}\right)^{1 / 6}$, where $\mathrm{n}$ is the solar wind particle density and $v$ its velocity. If the solar wind extends to Jupiter, the flux $\mathrm{n} v$ must be inversely proportional to the square of the distance from the sun. If we assume that the velocity is constant, the particle density at Jupiter is down by a factor of $(1 / 5.2)^{2}$ from that at Earth. Thus the ratio of magnetopause distances in units of planetary radii will be

$$
\frac{r_{\text {Jupiter }}}{r_{\text {Earth }}}=\left(5.2 \times \frac{10}{0.31}\right)^{1 / 3}=5.5
$$

For the calculations in this paper we shall assume a Jovian magnetopause at $50 R_{J}$ in the solar direction. The magnetospheric cross section as viewed from the sun or Earth would be about $200 \mathrm{R}_{\mathrm{J}}$ in diameter. Jupiter's magnetosphere at opposition would therefore have an apparent diameter in the sky of about $1.3^{\circ}$ and an apparent cross section area about seven times as great as the sun or moon.

The magnetospheric plasma and associated trapped radiation co-rotate with the planet out to about $50 \mathrm{R}_{\mathrm{J}}$ (Carr and Gulkis, 1969; Brice and Ioannidis, 1970). This conclusion is reached from an analysis of the formation of the Earth's plasmapause, which marks the boundary of the co-rotating portion of the magnetosphere (Brice, 1967). The plasmapause occurs where the convective electric field is about equal tothe co-rotation electric field. Whereas at Earth they are equal at about $5 R_{E}$, on Jupiter the co-rotation electric field is everywhere orders of magnitude greater than the convective field (Brice and Ioannidis, 1970). We therefore expect to find co-rotation of trapped particles all the way out to Jupiter's magnetopause. 


\section{CHARACTERISTICS OF JUPITER'S INNER SATELLITES}

Jupiter has twelve known satellites. Of these, the five inner ones might be expected to have a significant effect on its trapped radiation. These consist of the four Galilean satellites, large enough to be seen even with small telescopes, plus the small satellite JV, sometimes referred to as Amalthea, nearest to Jupiter. The remaining seven satellites are very small, much further away from Jupiter, and are in highly inclined and elliptical orbits.

The basic characteristics of the five inner satellites are given in Table 1. All five are in almost precise circular orbits with zero inclination. L-values at the position of each satellite are calculated, using a Jovian equatorial radius $R_{J}$ of $70,850 \mathrm{~km}$ (Dollfus, 1970a). Due to the large oblateness of Jupiter, these L-values would be about $2.2 \%$ larger if a mean radius $(69,320 \mathrm{~km})$ were used instead of the equatorial radius. Figure 2 shows the position of these satellites with respect to Jupiter and its magnetosphere.

Also calculated is the co-rotation period $\tau_{c r}$ of each satellite. This is the apparent (retrograde) period in a frame of reference rotating with Jupiter's decametric rotation period, $9^{\mathrm{h}} 55^{\mathrm{m}} 29.73^{\mathrm{s}}$ (Donivan and Carr, 1969). The decametric period is believed to be associated with Jupiter's solid surface and internal magnetic field; it is used to define Jupiter's System III longitude system. The satellites' System III co-rotation period will be important in a later section where we analyze the effect of satellite motion relative to trapped particles co-rotating with the decametric period. 
TABLE 1

Characteristics of Jupiter's five inner satellites. The radial distance, sidereal period, inclination and orbital eccentricity are taken from Blanco and McCuskey (1961). $L$ is the radial distance in units of one Jupiter equatorial radius, $R_{J}=$ $70,850 \mathrm{~km}$ (Dollfus, 1970a). The co-rotation period $\tau_{\mathrm{cr}}$ is the apparent (retrograde) period of the satellite in a frame of reference rotating with the decametric rotation period, 0.413539 days (Donivan and Carr, 1969). The satellite diameters are taken from Dollfus (1970b).

SATELLITE

\begin{tabular}{lccccc} 
& $\begin{array}{c}\text { JV } \\
\text { Amalthea }\end{array}$ & $\begin{array}{c}\text { JI } \\
\text { Io }\end{array}$ & $\begin{array}{c}\text { JII } \\
\text { Europa }\end{array}$ & $\begin{array}{c}\text { JIII } \\
\text { Ganymede }\end{array}$ & $\begin{array}{c}\text { JIV } \\
\text { Callisto }\end{array}$ \\
\cline { 2 - 6 } $\begin{array}{l}\text { Radial } \\
\text { distance, kms }\end{array}$ & 180,500 & 421,600 & 670,800 & $1,070,000$ & $1,882,000$ \\
L & 2.55 & 5.95 & 9.47 & 15.10 & 26.56 \\
$\begin{array}{l}\text { Inclination } \\
0^{\circ} 24^{\prime}\end{array}$ & $0^{\circ} 2^{\prime}$ & $0^{\circ} 28^{\prime}$ & $0^{\circ} 11^{\prime}$ & $0^{\circ} 15^{\prime}$ \\
$\begin{array}{l}\text { Eccentricity } \\
\begin{array}{l}\text { Sidereal } \\
\text { period, days }\end{array}\end{array}$ & 0.003 & 0.0000 & 0.0003 & 0.0015 & 0.0075 \\
$\begin{array}{l}\text { Co-rotation } \\
\text { period } \tau \text { cr } \text {, days }\end{array}$ & 0.498 & 1.769 & 3.551 & 7.155 & 16.689 \\
$\begin{array}{l}\text { Diameter } \\
D_{\text {s }}, \mathrm{km}\end{array}$ & 2.434 & 0.540 & 0.468 & 0.439 & 0.424 \\
\hline
\end{tabular}




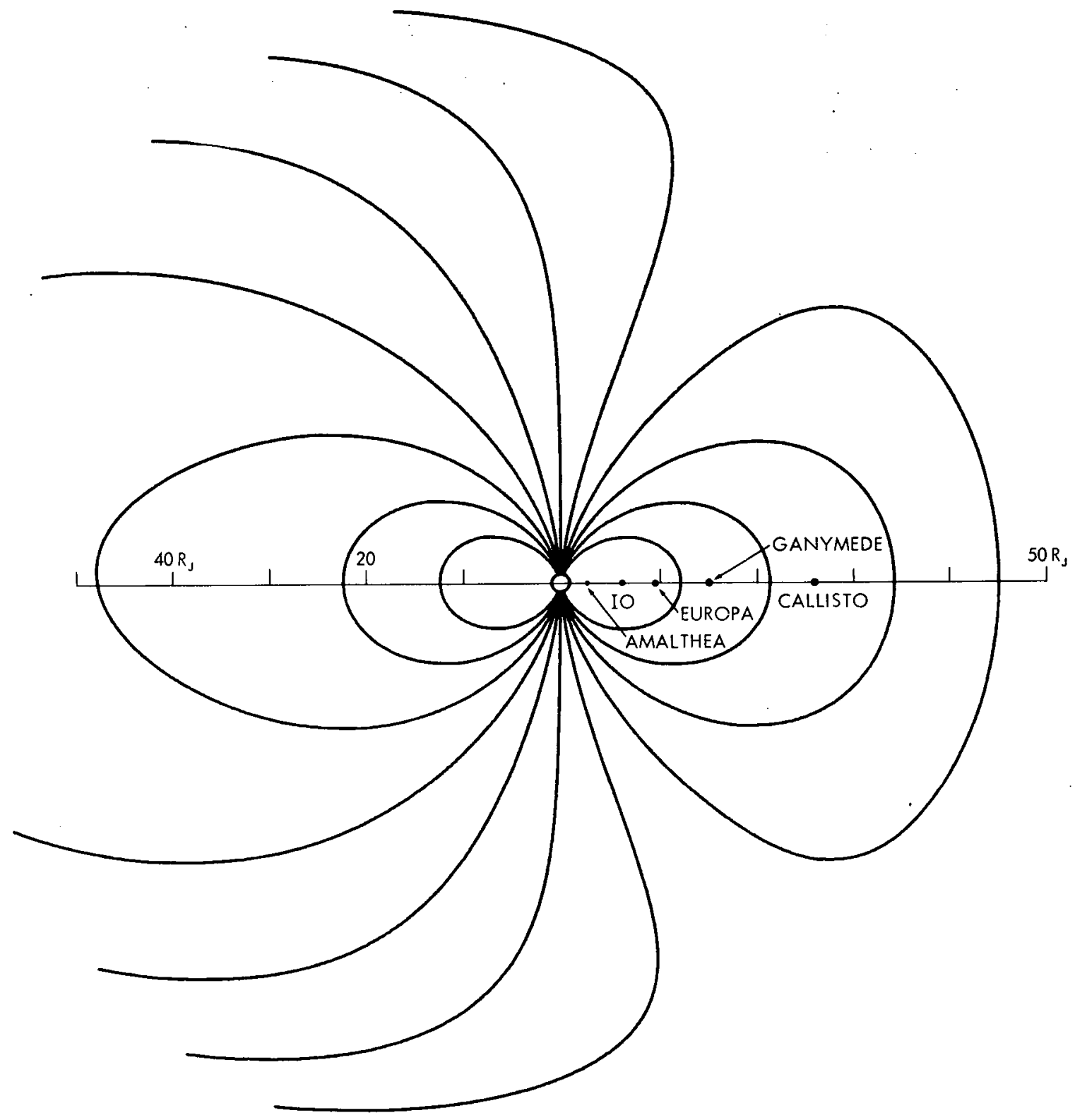

Figure 2. The position of Jupiter's five inner satellites in its magnetosphere. Diameters are not to scale. 
The diameter of Io is about the same as the Earth's moon, Europa is slightly smaller, and Ganymede and Callisto are about 50\% larger. Amalthea is very much smaller, but due to its innermost position it can significantly influence the radiation belts. 


\section{TRAPPED-PARTICLE MOTION AT JUPITER}

We now examine the behavior of trapped particles in Jupiter's magnetosphere. Weassume that particles co-rotate with the planet and that the equatorial magnetic field is $10 / \mathrm{L}^{3}$ gauss. Typical characteristics of near-equatorial protons and electrons near the surface $(\mathrm{L}=1)$ and near each of the five inner satellites are shown in Table 2. We assume conservation of the magnetic moment $\mu$. Thus particle energies increase with decreasing distance.

Nonrelativistically, the kinetic energy of a particle is given by $\mathbf{E}=\mu \mathbf{B}_{\mathrm{m}}$, where $B_{m}$ is the field magnitude at the mirror point. However, the electrons in the inner regions will be relativistic, and the relativistic form of the first invariant must be used:

$$
\mu=\mathrm{p}_{\perp}^{2} / 2 \mathrm{~m}_{0} \mathrm{~B}=\mathrm{p}^{2} / 2 \mathrm{~m}_{\sigma} \mathrm{B}_{\mathrm{m}}
$$

where $\mathrm{p}$ is the relativistic momentum and $\mathrm{m}_{0}$ is the rest mass. The kinetic energy is then given by

$$
E=\left(p^{2} c^{2}+m_{0}^{2} c^{4}\right)^{1 / 2}-m_{0} c^{2}
$$

In the extreme relativistic limit this reduces to

$$
E=\left(2 m_{0} c^{2} \mu B_{m}\right)^{1 / 2}-m_{0} c^{2}
$$

with $\mathrm{m}_{0} \mathrm{c}^{2}=0.511 \mathrm{Mev}$ for electrons. Thus, very roughly, $\mathrm{E}^{2}=\mu \mathrm{B}_{\mathrm{m}}$ for relativistic electrons, with energies in $\mathrm{Mev}$.

We have chosen a value of $50 \mathrm{Mev} /$ gauss for the magnetic moment of both protons and electrons as characteristic of typical particles which might become 


\section{TABLE 2}

Characteristics of near-equatorial protons and electrons near the surface of Jupiter $(L=1)$ and at the distance of each of the five inner satellites, assuming a magnetic moment $\mu=50 \mathrm{Mev} /$ gauss and an equatorial surface field at Jupiter of 10 gauss. $E$ is the kinetic energy, $R_{c}$ is the cyclotron radius, $\tau_{b}$ is the bounce period, and $\tau_{\mathrm{d}}$ is the drift period.

\begin{tabular}{|c|c|c|c|c|c|c|}
\hline & $\mathrm{L}=1$ & Amalthea & Io & Europa & Ganymede & Callisto \\
\hline $\mathrm{L}$ & 1.00 & 2.55 & 5.95 & 9.47 & 15.10 & 26.56 \\
\hline $\mathrm{B}$, gauss & 10.00 & 0.6048 & 0.0475 & 0.0118 & 0.0029 & 0.00053 \\
\hline \multicolumn{7}{|l|}{ Protons } \\
\hline $\mathrm{E}, \mathrm{Mev}$ & 410 & 29.8 & 2.37 & 0.589 & 0.145 & 0.027 \\
\hline $\mathrm{R}_{\mathrm{c}}, \mathrm{km}$ & 3.2 & 13.1 & 46.9 & 94.1 & 190 & 442 \\
\hline$\tau_{b}$, mins & 0.016 & 0.122 & 0.991 & 3.16 & 10.1 & 41.6 \\
\hline$\tau_{\mathrm{d}}$, days & 0.35 & 1.63 & 8.65 & 21.8 & 55.6 & 172 \\
\hline \multicolumn{7}{|l|}{ Electrons } \\
\hline $\mathrm{E}, \mathrm{Mev}$ & 22.1 & 5.07 & 1.128 & 0.418 & 0.129 & 0.026 \\
\hline $\mathrm{R}_{\mathrm{c}}, \mathrm{km}$ & 0.075 & 0.31 & 1.09 & 2.20 & 4.42 & 10.3 \\
\hline$\tau_{b}, \operatorname{mins}$ & 0.012 & 0.030 & 0.074 & 0.13 & 0.30 & 1.02 \\
\hline$\tau_{\mathrm{d}}$, days & 10.8 & 17.3 & 27.7 & 39.7 & 69.6 & 181 \\
\hline
\end{tabular}


trapped in Jupiter's outer magnetosphere. As indicated in Table 2, maximum equatorial proton energies for this value of $\mu$ are about $400 \mathrm{Mev}$. Electron energies in the heart of the synchrotron emission region are about $10 \mathrm{Mev}$, consistent with earlier estimates. Brice (1972) estimates that protons inside Jupiter's bow shock would have a magnetic moment of about $100 \mathrm{Mev} /$ gauss, and electrons about $20 \mathrm{Mev} / \mathrm{gauss}$.

Note that if the first invariant is conserved, the kinetic energy of relativistic equatorial electrons varies as $L^{-1.5}$, whereas the energy of nonrelativistic protons varies as the usual $\mathrm{L}^{-3}$. Protons are therefore much more energetic than electrons with the same magnetic moment in the inner regions, where for electrons $\mu \mathrm{B} \gg \mathrm{m}_{0} \mathbf{c}^{2}$.

Shown also in Table 2 are the cyclotron radius $R_{c}$, the bounce period $\tau_{b}$, and the drift period $\tau_{d}$ in the co-rotating frame for near-equatorial particles, as calculated from the following formulas (Hess, 1968), applicable to particles of any energy:

$$
\begin{gathered}
\mathrm{R}_{\mathrm{c}}=\mathrm{pc} / \mathrm{eB} \\
\tau_{\mathrm{b}}=3 \mathrm{~L} \mathrm{R}_{\mathrm{J}} / \mathrm{v} \\
\tau_{\mathrm{d}}=4 \pi \mathrm{e} \mathrm{B}_{0} \mathrm{R}_{\mathrm{J}}^{2} / 3 \mathrm{~L} \mathrm{~m}_{0} \mathrm{v}^{2} \mathrm{c} \gamma
\end{gathered}
$$

where $\mathrm{v}$ is the particle velocity and $\mathrm{B}_{0}$ is the surface equatorial field (10 gauss). The drift period scales as $B_{0} R^{2}$, where $R$ is the planetary radius, and therefore is several orders of magnitude larger on Jupiter than on Earth. In Jupiter's field, the drift period for near-equatorial nonrelativistic protons or electrons in the co-rotating frame simplifies to $\tau_{d}=122 / \mathrm{LE}$ days, with $\mathrm{E}$ in Mev. The 
drift period for highly relativistic particles is a factor of two greater. For nonequatorial particles $\tau_{d}$ is multiplied by an additional factor $F(\lambda)$, ranging from 1.0 to 1.5 , where $\lambda$ is the mirror latitude. The bounce period for non-equatorial particles is also multiplied by a factor ranging from 1.0 to 1.9 , depending on the mirror latitude. Thus the values given in the table are valid within a factor of two for non-equatorial particles as well.

Nonrelativistically, $\mathbf{R}_{\mathrm{c}} \propto \mu^{1 / 2}, \tau_{\mathrm{b}} \propto \mu^{-1 / 2}$, and $\tau_{\mathrm{d}} \propto \mu^{-1}$. In the extreme relativistic limit $\mathbf{R}_{c} \propto \mu^{1 / 2}, \tau_{b}$ is constant, and $\tau_{d} \propto \mu^{-1 / 2}$. Thus the values given in Table 2 are not strongly dependent on the specific value we have chosen for the magnetic moment.

A comparison of Tables 1 and 2 leads to the following conclusions:

1. Cyclotron radii are small compared with satellite diameters. Thus we need consider only the motion of the guiding center as we study the interaction of trapped particles with Jupiter's satellites.

2. Drift periods in the co-rotating frame are very long on Jupiter, and co-rotation dominates. Jupiter's energetic trapped particles thus behave very differently from those at Earth, where drift periods are typically short compared with the 24-hour rotation period.

We can now form a picture of particle motion in the vicinity of the five inner satellites. Since the relative motions are fairly complex, one can easily reach erroneous conclusions unless one thinks in terms of the most appropriate coordinate system. We have found that the best system to visualize is a frame of reference as shown in Figure 3. This system is centered on the magnetic dipole, 
with its $\mathrm{z}$-axis aligned with the dipole, and co-rotating with the planet. The axes are fixed with respect to the solid planet and define the jovimagnetic dipole coordinate system. In this system the low-energy plasma appears to be stationary, and high-energy trapped particles drift in longitude with periods of the order of weeks. Jupiter is stationary, and its satellites appear to be in retrograde orbits with an inclination of $10^{\circ}$ with respect to the equator and periods given by the co-rotation period in Table 1, i.e., about half a day except for Amalthea. The satellite orbits, although tilted, are coplanar, with their common nodes defined by the intersection of the jovimagnetic equator with the rotational equator. These nodes do not precess. The satellites appear to differ only in their radial distance and their apparent rate of retrograde motion. Due to the offset of the dipole, the satellite orbits, although circular, appear to be offset by an equal amount; perijove is perhaps $0.2 R_{J}$ closer than apojove. Perijove, however, is always at the same jovimagnetic longitude for all satellites. The plane in which the satellites appear to revolve is perhaps as much as $0.1 R_{J}$ above or below the jovimagnetic equator, in addition to being tilted by $10^{\circ}$.

In the next section we shall use a simplified version of this picture, where we ignore the effects of dipole inclination, dipole offset, quadrupole and higherorder terms, and distortion of Jupiter's magnetosphere topology by the solar wind. In later sections we will examine the consequences of removing some of these simplifying assumptions. 


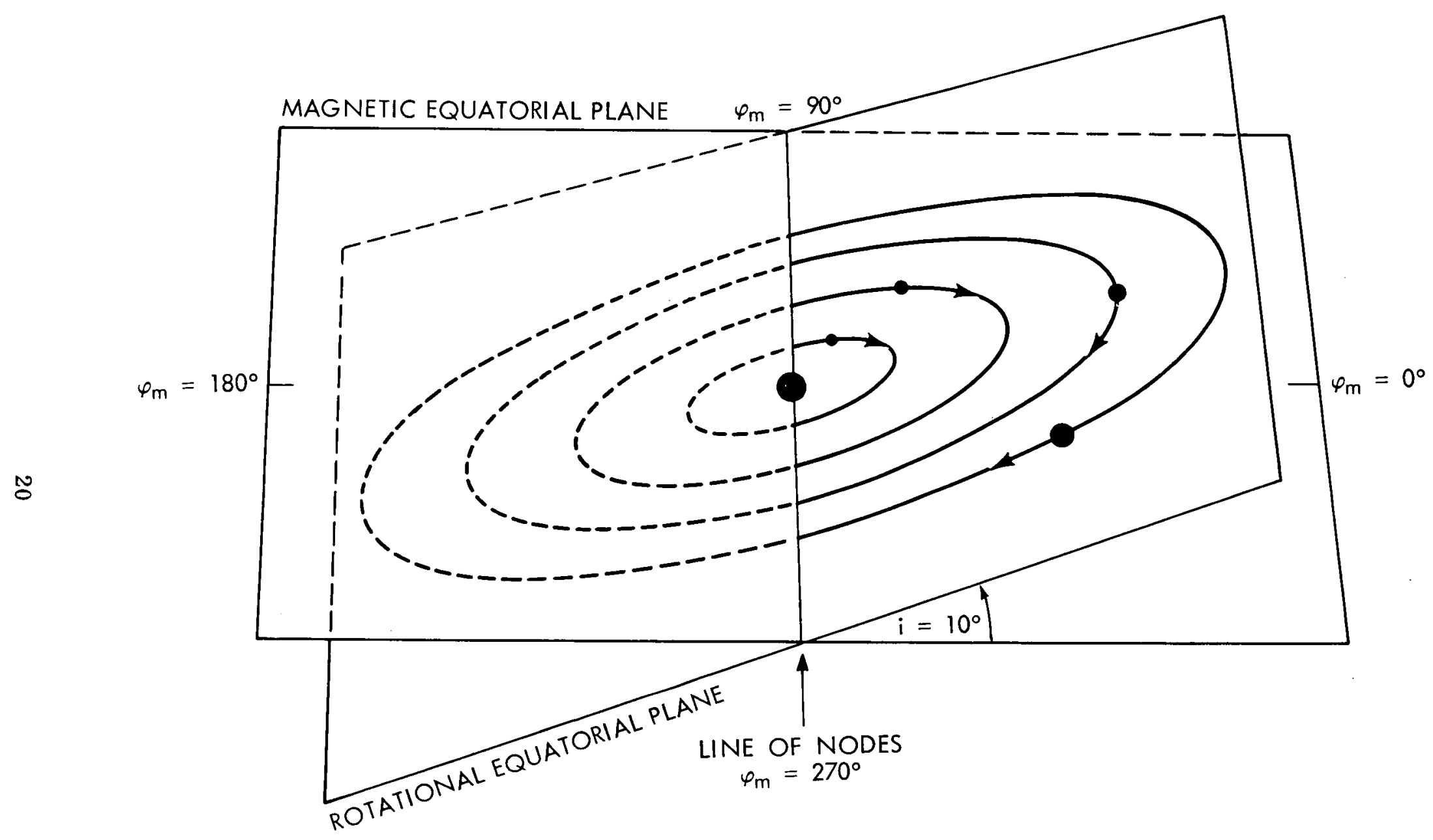

Figure 3. Co-rotating jovimagnetic coordinate system. Jupiter and the thermal plasma are stationary in this system. The inner satellites appear to revalve in a retrograde direction with an inclination of $10^{\circ}$ and periods of about $10-13$ hours (60 hours for Amalthea). 


\section{SWEEPING EFFECT OF JUPITER'S SATELLITES}

The possibility that a planetary satellite might sweep out a region of a radiation belt was first considered by Singer (1962). At the time it was thought that Mars had a magnetic field and a radiation belt similar to the Earth's. Singer calculated the sweeping effect of the tiny satellite Phobos, located at a martian $\mathrm{L}=2.8$. In this section we calculate the rate at which near-equatorial trapped particles are removed by Jupiter's inner satellites. To simplify the analysis, we make the following assumptions:

1. The dipole is centered and aligned with the rotational axis.

2. The field is purely dipolar - i.e., no quadrupole or higher-order effects and no external distortions.

3. The particles' guiding-center motion is not affected by the nearby presence of a satellite. Thus we exclude the possibility that a particle might be deflected by the electric or magnetic field surrounding a satellite. If in its normal motion a particle would impact a satellite, we assume that the particle will be removed from the radiation belt. This is the most crucial assumption, and if it is not true, the major conclusions of this study would be substantially altered.

As in the previous section, we assume that all trapped particles co-rotate with the planet. In our co-rotating reference frame the satellites will appear to revolve in the equatorial plane with retrograde co-rotation periods $\tau_{\mathrm{cr}}$ given by Table 1. As the particles bounce back and forth through the equator, they have a finite probability of impacting a satellite. In the analysis we distinguish between two cases, depending upon the longitudinal distance $d$ that a satellite appears 
to move with respect to the trapped particles during one half bounce period. This distance is given by

$$
\mathrm{d}=\pi \mathrm{L} \mathrm{R}_{\mathrm{J}} \tau_{\mathrm{b}} / \tau_{\mathrm{cr}}
$$

If this distance is greater than a satellite diameter $D_{s}$ (case 1), the absorption is a random process; in one apparent revolution of the satellite only some fraction of the particles at the position of the satellite will be absorbed. On the other hand, if $d$ is less than a satellite diameter (case 2), the satellite will act like a snowplow and remove all particles in its path.

Case 1: Random absorption ( $d>D_{s}$ ). This case was considered by Mead (1972). We consider a narrow ring of monoenergetic trapped particles whose radial width in the equatorial plane is exactly the diameter of the satellite $D_{s}$ (Figure 4). For the moment we assume no diffusion of particles into or out of the ring. In one half bounce period each particle will pass through the equatorial plane once. Those which happen to impact the satellite will be removed. One half bounce period later the satellite will appear to have moved in longitude by at least one satellite diameter; therefore, it will absorb a whole new group of particles. Many of the particles in between will be missed, but these will have a finite probability of being absorbed the next time around.

In one half bounce period, $\tau_{b} / 2$, the average probability $P$ that any one particle in the ring will be absorbed is therefore given by the ratio of the absorption cross section of the satellite $\left(\pi \mathrm{D}_{\mathrm{s}}^{2} / 4\right)$ to the area of the ring $(2 \pi \mathrm{L}$ $R_{J} D_{s}$; i.e.,

$$
P=D_{s} / 8 L R_{J}
$$




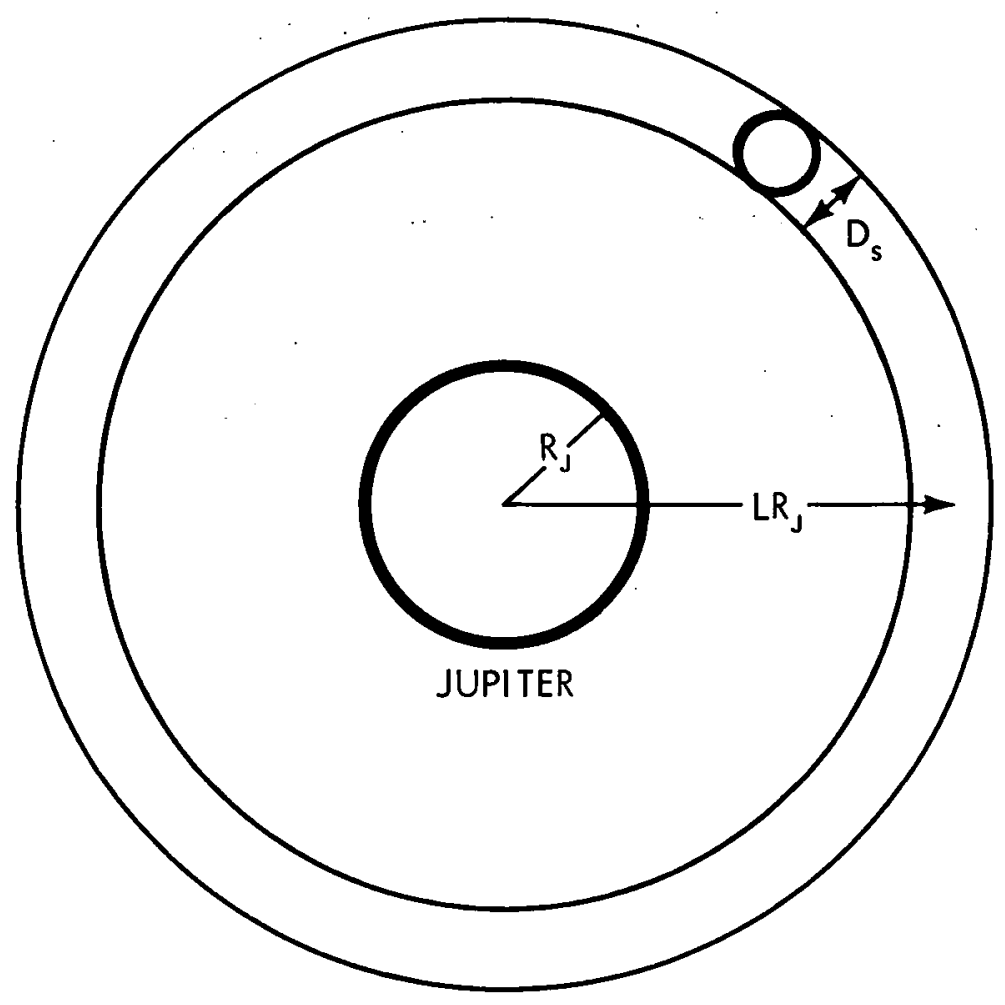

Figure 4. Sketch used to calculate absorption and diffusion times near a satellite with diameter $D_{s}$. 
The fraction lost per unit time is then given by $2 \mathrm{P} / \tau_{\mathrm{b}}=\mathrm{D}_{\mathrm{s}} / 4 \tau_{\mathrm{b}} \mathrm{L} \mathrm{R}_{\mathrm{J}}$, and over many co-rotation periods the mean absorption lifetime $\tau_{\text {abs }}$ before impacting the satellite is given by the inverse of this quantity, i.e.,

$$
\tau_{\text {abs }}=\tau_{\mathrm{b}} / 2 \mathrm{P}=4 \tau_{\mathrm{b}} \mathrm{L} \mathrm{R}_{\mathrm{J}} / \mathrm{D}_{\mathrm{s}} \quad(\text { Case } 1)
$$

Since $\tau_{b} \alpha^{\cdot} \mu^{-1 / 2}$ for nonrelativistic particles, the Case 1 absorption lifetime varies as the inverse square root of the magnetic moment.

Case 2: Snowplow absorption $\left(d<D_{s}\right)$. If the apparent drift of the satellite in one half bounce period is less than a satellite diameter, all particles in the center of the ring (Figure 4) will be absorbed in one corotation period. Although some near the edge of the ring might slip past, the mean lifetime $\tau_{a b s}$ will be approximately equal to the co-rotation period:

$$
\tau_{\mathrm{abs}} \approx \tau_{\mathrm{cr}}(\text { Case } 2)
$$

The Case 2 absorption lifetime is independent of the magnetic moment of the particle. Except for a factor of $\pi / 4$, the Case 1 and 2 absorption lifetimes are equal for $d=D_{s}$.

In Table 3 values of $d$ are given for electrons and protons with magnetic moment $\mu=50 \mathrm{Mev} /$ gauss at the position of each of the satellites. It is clear that for protons, Case 1 applies at Europa, Ganymede, and Callisto, and Case 2 applies elsewhere. For electrons, which bounce through the equator much more frequently due to their greater velocity, Case 1 applies at Callisto, but Case 2 applies elsewhere. The values of the mean lifetime before absorption $\tau_{\text {abs }}$ are given in Table 3 and plotted in Figure 5 for electrons and protons, with the 


\section{TABLE 3}

Comparison of absorption and diffusion rates for particles with $\mu=50 \mathrm{Mev} /$ gauss. The parameter $d$ is the longitudinal distance each satellite appears to move with respect to the co-rotating system during one half bounce period; it determines whether Case 1 or Case 2 applies. $\tau_{\text {abs }}$ is the mean lifetime before absorption and $\tau_{\mathrm{diff}}$ is a characteristic diffusion time near each satellite. $\Delta r$ is the inward distance that an equatorial particle attached to a field line would move during a sudden commencement which moved the boundary in from 50 to $40 R_{J} \cdot F$ is the fraction of the particles which survive absorption in diffusing past each satellite.

\section{Satellite}

\begin{tabular}{|c|c|c|c|c|c|}
\hline . & Amalthea & Io & Europa & Ganymede & Callisto \\
\hline $\mathrm{d}$, protons & $20 \mathrm{~km}$ & $1700 \mathrm{~km}$ & $9900 . \mathrm{km}$ & $54,000 \mathrm{~km}$ & $403,000 \mathrm{~km}$ \\
\hline d, electrons & $5 \mathrm{~km}$ & $125 \mathrm{~km}$ & $420 \mathrm{~km}$ & $1600 \mathrm{~km}$ & $9900 \mathrm{~km}$ \\
\hline$\tau_{\text {abs }}$, protons & $2.43 \mathrm{~d}$ & $0.54 \mathrm{~d}$ & $1.90 \mathrm{~d}$ & $5.43 \mathrm{~d}$ & $43.5 \mathrm{~d}$ \\
\hline$\tau_{\text {abs }}$, electrons & $2.43 \mathrm{~d}$ & $0.54 \mathrm{~d}$ & $0.47 \mathrm{~d}$ & $0.44 \mathrm{~d}$ & $1.07 \mathrm{~d}$ \\
\hline \multicolumn{6}{|c|}{ Magnetic diffusion } \\
\hline$\dot{\tau}_{\text {diff }}$ & $137 \mathrm{yr}$ & $8.7 \mathrm{yr}$ & $24 \mathrm{~d}$ & $0.7 \mathrm{~d}$ & $0.002 \mathrm{~d}$ \\
\hline$\Delta \mathbf{r}$ & $10 \mathrm{~km}$ & $308 \mathrm{~km}$ & $2090 \mathrm{~km}$ & $14,800 \mathrm{~km}$ & $165,000 \mathrm{~km}$ \\
\hline $\mathrm{F}$, protons & $10^{-125}$ & $10^{-66}$ & 0.0016 & 0.788 & 0.999 \\
\hline electrons & $10^{-125}$ & $10^{-66}$ & $1.2 \mathrm{E}-6$ & 0.159 & 0.996 \\
\hline
\end{tabular}

Electric diffusion

\begin{tabular}{lccccc}
$\tau_{\text {dif f }}$ & $208 \mathrm{~d}$ & $393 \mathrm{~d}$ & $19 \mathrm{~d}$ & $3.7 \mathrm{~d}$ & $0.1 \mathrm{~d}$ \\
F, protons & $10^{-8}$ & $10^{-23}$ & 0.0036 & 0.370 & 0.995 \\
F, electrons & $10^{-8}$ & $10^{-23}$ & $6.0 \mathrm{E}-6$ & 0.006 & 0.838 \\
\hline
\end{tabular}




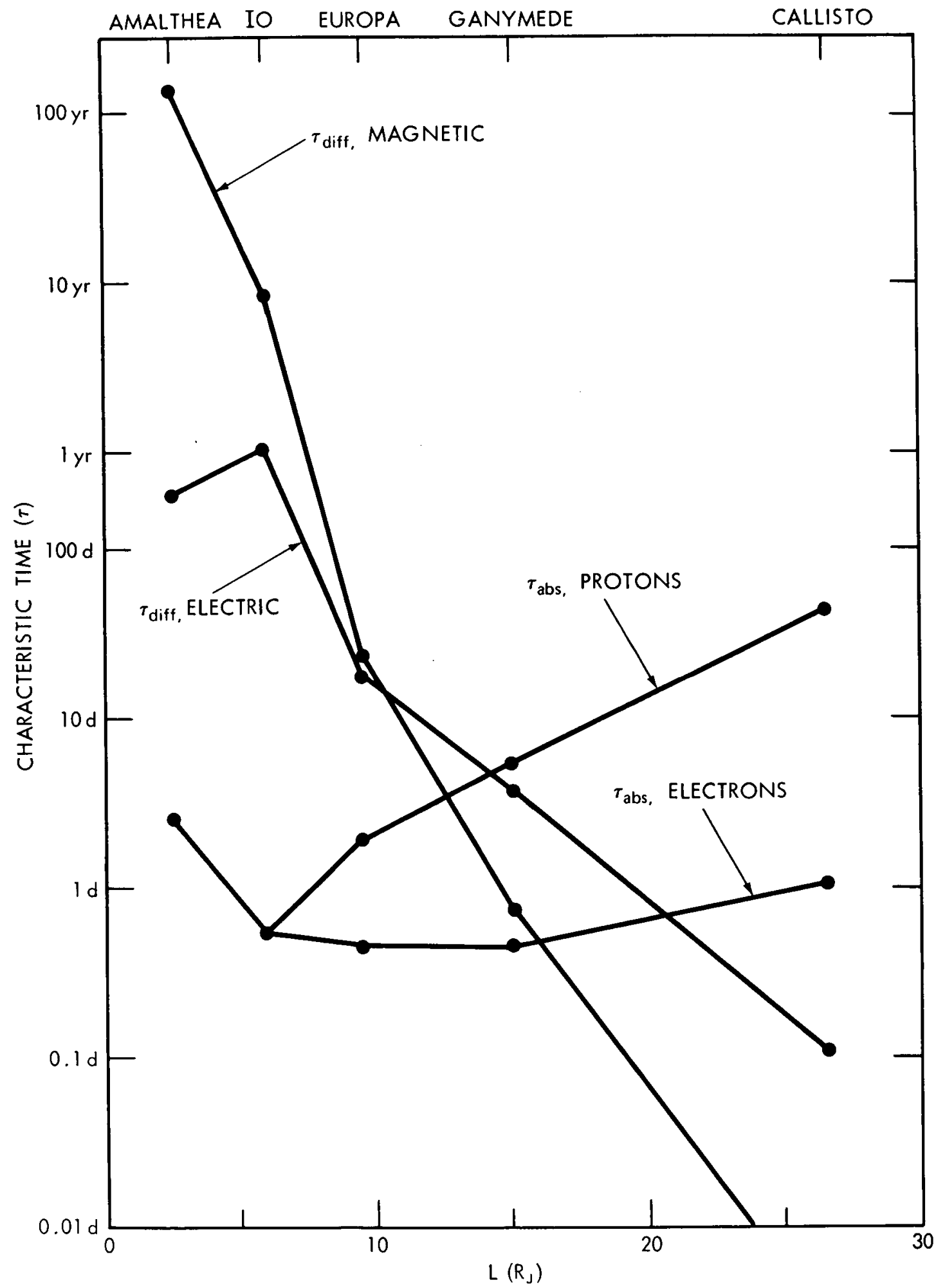

Figure 5. Characteristic absorption and diffusion times near each satellite. Diffusion dominates near Callisto, but absorption dominates of Amalthea, Io, and Europa. 
appropriate Case 1 or 2 formula being used. These lifetimes are of the order of a few days, except for protons near Callisto. The values are rather insensitive to the magnetic moment of the particles.

There will be a group of particles mirroring at latitudes less than $10^{\circ}$ (equatorial pitch angles greater than $69^{\circ}$ ) that will not strike the satellites as often because of the $10^{\circ}$ tilt between the magnetic axis and the rotational axis (see Figure 3). The mean absorption lifetime of these particles will be considerably longer than for the particles which mirror at higher latitudes. We will consider these particles in a later section.

Whether or not particles can remain in the radiation belts, despite their being continually swept out by the satellites, depends on how rapidly radial diffusion moves them past the orbit of the satellites, and, in the case of electrons, whether additional source mechanisms are present. In the next section we attempt to compare rates of radial diffusion with rates of absorption by the satellites. 


\section{RADIAL DIFFUSION RATES AT JUPITER}

In an earlier section we argued that radial diffusion by violation of the third invariant was likely to be the main mechanism for moving solar-wind protons into the inner regions of Jupiter's magnetosphere. Their energy increases with decreasing distance from the planet if the first two invariants are conserved. Trapped electrons would also undergo radial diffusion. However, a number of additional processes, such as pitch-angle diffusion and local acceleration mechanisms, are likely to affect the electrons. The diffusion analysis in this section is therefore expected to apply primarily to protons, which we believe should behave in predictable ways. The behavior of electrons, as at Earth, is likely to be much more complex.

The most difficult part of any trapped-particle diffusion analysis is to estimate the value of the diffusion coefficient $D$, which governs the rate of diffusion in different regions of the magnetosphere. At Earth both theoretical and experimental techniques have been used to estimate D. In recent reviews, Walt $(1971 \mathrm{a}, \mathrm{b})$ has summarized the present status of radial diffusion studies. Almost all the studies indicated a diffusion coefficient increasing extremely rapidly with $\mathrm{L}$. The theoretical studies led to a coefficient proportional to $\mathrm{L}^{10}$ or $\mathrm{L}^{6}$, depending upon whether magnetic disturbances or electric potential disturbances were considered to be the dominant diffusing force. The limited experimental studies generally could not determine the exact dependence upon L, except for one study where D was found to vary as $L^{10 \pm 1}$ (Newkirk and Walt, 1968). There was in general a scatter of several orders of magnitude at all L-values in the estimated value of $D$. This was understandable, considering the variety 
of ways in which the values were obtained, and the fact that both proton and electron studies were considered together. In general, the various analyses have not considered the possible dependence of D upon the particle energy or species. One exception is the recent theoretical analysis by Cornwall (1972) of the radial diffusion of ionized helium and protons, where the dependence of the electrostatic radial diffusion coefficient upon the charge $Z$, mass number $A$, and energy was specifically considered.

At Jupiter the problem of estimating $\mathrm{D}$ is even more difficult. We have only indirect estimates of electron energies and intensities, and no experimental information on protons. We have almost no way of obtaining quantitative estimates of fluctuating magnetic and electric fields, except to assume that solar wind fluctuations at Jupiter are similar to those at Earth. In this section we take a very simplified approach to the determination of diffusion rates at Jupiter. We treat only equatorial particles, and we assume that $D$ is independent of particle energy. We attempt to estimate a magnetic diffusion coefficient, using an approach similar to that taken by Nakada and Mead (1965) in their study of diffusion of protons in the Earth's radiation belt. We then compare typical magnetic diffusion times in the vicinity of each satellite with the absorption times calculated in the previous section, to see whether particles can diffuse past the satellite before they are absorbed. And finally, we examine the possible effects of an $L^{6}$-type diffusion, as might be produced by electric field fluctuations.

Magnetic diffusion. Here we estimate the rate of radial diffusion at Jupiter due to magnetic fluctuations associated with fluctuations in the solar wind. We restrict our analysis to equatorially-trapped particles. As in Nakada and Mead 
(1965), we assume violation of the third invariant associated with magnetic disturbances caused by sudden increases in the solar wind intensity, followed by a gradual relaxation of the magnetosphere, during which the third invariant is conserved.

We assume that the distorted field strength in the equatorial plane is given by

$$
B=\frac{M}{r^{3}}+\frac{a_{1}}{r_{b}^{3}}+\frac{a_{2}}{r_{b}^{4}} r \cos \phi
$$

where $M$ is the surface equatorial field strength (10 Gauss), $r$ is the distance in jovian radii, $r_{b}$ is the distance to the magnetopause in the solar direction, $\phi$ is the solar magnetic longitude ( $\phi=0$ at magnetic local noon), and from Mead's (1964) model $\mathrm{a}_{1}=0.816 \mathrm{M}$ and $\mathrm{a}_{2}=0.673 \mathrm{M}$.

Near Jupiter, the quiet-time contribution to the total field from the magnetopause currents is given by $a_{1} / r_{b}^{3}$, where $r_{b}$ is the quiet-time boundary distance, which we assume to be $50 R_{J}$. If a sudden increase in the solar wind intensity moves the boundary in to a new position $r_{c}$ and the magnetosphere subsequently relaxes slowly, the mean square radial displacement of a particle at a distance $r$ is given by

$$
\left\langle(\Delta r)^{2}\right\rangle=\frac{25}{98}\left(\frac{a_{2} r^{5}}{M}\right)^{2}\left(\frac{1}{r_{c}^{4}}-\frac{1}{r_{b}^{4}}\right)^{2}
$$

(Nakada and Mead, 1965, Equation 3).

We now assume that on the average there is one disturbance per (Earth) day which doubles the contribution of the external field near Jupiter, i.e., 


$$
a_{1} / r_{c}^{3}=2 a_{1} / r_{b}^{3}
$$

This is equivalent to a doubling of the quantity $n^{1 / 2} v$ at Jupiter, where $n$ is the solar wind density and $\mathrm{v}$ is its velocity. Then the resulting average diffusion coefficient $\mathrm{D}$ is given by

$$
\begin{aligned}
D & =\frac{1}{2}\left\langle\frac{(\Delta r)^{2}}{\Delta t}\right\rangle=\frac{25}{196}\left(\frac{a_{2}}{M}\right)^{2}\left(\frac{r^{10}}{r_{b}^{8}}\right)\left(2^{4 / 3}-1\right)^{2} \\
& =0.13 \mathrm{r}^{10} / \mathrm{r}_{\mathrm{b}}^{8} \mathrm{day}^{-1} \\
& =3.4 \times 10^{-15} \mathrm{~L}^{10} \mathrm{R}_{\mathrm{J}}^{2} / \mathrm{day} \\
& =2.0 \times 10^{-10} \mathrm{~L}^{10} \mathrm{~km}^{2} / \mathrm{s} \mathrm{ec}
\end{aligned}
$$

taking $r_{b}=50 R_{J}$

In Nakada and Mead (1965) the diffusion coefficient was determined by counting the number of sudden commencements and sudden impulses of varying sizes observed per year. This led to a value of $D$ equal to $0.0155 \mathrm{r}^{10} / \mathrm{r}_{\mathrm{b}}^{8} \mathrm{day}^{-1}$, or $1.55 \times 10^{-10} \mathrm{~L}^{10} \mathrm{R}_{\mathrm{E}}^{2} /$ day, taking $\mathrm{r}_{\mathrm{b}}=10 \mathrm{R}_{\mathrm{E}}$. Thus if an analysis similar to the present one had been used in that paper (one sudden doubling of the solar wind velocity per day), the resulting diffusion coefficient would have been about ten times as large, and roughly equal to the value quoted by Williams (1972) as that value which could explain the behavior of outer-belt protons. Nakada and Mead (1965) indicated that an increase in their diffusion coefficient by a factor of 8 would have yielded better agreement with experimentally-measured outer-belt proton fluxes, and Fälthammar (1966) suggested that consideration of the effect of a large number of small, nearly continuous field fluctuations could give the 
required increase in D. In the present study, therefore, although we have probably overestimated the frequency of large disturbances at Jupiter, consideration of a spectrum of magnetic disturbances would very likely lead to a value of $\mathrm{D}$ similar to the value used here.

We now wish to compare these diffusion rates with the loss rates due to absorption by the satellites. The simplest way is to calculate a characteristic diffusion time in the vicinity of each of the satellites and compare this with the mean lifetime before impact calculated in the previous section.

A characteristic diffusion time can be defined as follows. The one-dimensional Fokker-Planck equation for radial diffusion of trapped particles by violation of the third invariant is given by

$$
\frac{\partial n}{\partial t}=\frac{1}{2} \frac{\partial}{\partial r}\left[\left\langle\frac{(\Delta r)^{2}}{\Delta t}\right\rangle \frac{1}{r^{2}} \frac{\partial}{\partial r}\left(n r^{2}\right)\right]+S(r, t)
$$

(Birmingham et al., 1967, Equation 2.4), where $n(r, t)$ is the ensemble-averaged number of particles in the range $r$ to $r+d r$ at time $t$, and $S$ is the source and/or sink function describing injection and losses. Let us assume that in the immediate vicinity of the satellite $S=0$ and that $D$ is independent of $r$. The equation is then

$$
\begin{aligned}
\frac{\partial \mathrm{n}}{\partial \mathrm{t}} & =D \frac{\partial}{\partial \mathrm{r}}\left[\frac{1}{\mathrm{r}^{2}} \frac{\partial}{\partial \mathrm{r}}\left(\mathrm{n} \mathrm{r}^{2}\right)\right] \\
& =D \frac{\partial}{\partial \mathrm{r}}\left(\frac{\partial \mathrm{n}}{\partial \mathrm{r}}+\frac{2 \mathrm{n}}{\mathrm{r}}\right)
\end{aligned}
$$


We now assume that at time $t=0$ there are $n_{0}$ particles all located at a distance $r=r_{0}$. So long as the width of the evolving distribution function is narrow compared with the distance $r_{0}, \partial n / \partial r \gg 2 n / r$, and thus

$$
\frac{\partial \mathrm{n}}{\partial \cdot \mathrm{t}}=\mathrm{D} \frac{\partial^{2} \mathrm{n}}{\partial \mathrm{r}^{2}}
$$

which is in the form of the familiar diffusion equation. The solution satisfying the initial boundary condition is

$$
n(r, t)=\frac{n_{0}}{(4 \pi D t)^{1 / 2}} \exp \left(-\left(r-r_{0}\right)^{2 / 4 D t}\right)
$$

which can be verified by substitution into Equation 19 . Equation 20 describes the early evolution of a delta function of $n_{0}$ particles at $r=r_{0}, t=0$ into a gaussian of increasing width.

We now suppose that the $n_{0}$ particles are just at the outer edge of the ring of width $D_{s}$ (see Figure 4). We then ask how long it will take for $n(r, t)$ to develop into a gaussian whose value at the inner edge of the ring $\left(r=r_{0}-D_{s}\right)$ is just $1 / \mathrm{e}$ of its maximum value at the outer edge. At the inner edge

$$
\left(r-r_{0}\right)^{2} / 4 \mathrm{Dt}=1
$$

and therefore

$$
\tau_{\text {diff }}=D_{s}^{2} / 4 D
$$

The calculated values of this characteristic diffusion time at each of the five inner satellites are given in Table 3 and plotted in Figure 5. It is evident that 
the diffusion times are long compared with the absorption times at Amalthea, Io, and Europa. The extreme variation from Amalthea to Callisto is due, of course, to the $\mathrm{L}^{10}$ dependence of the diffusion coefficient.

The fraction of particles that can diffuse in past a satellite without being absorbed can be calculated by obtaining a steady-state solution to the FokkerPlanck equation in the region of satellite absorption. We assume that the rate of absorption is constant throughout the ring within which the satellite moves (see Figure 4) and can be characterized by an average absorption time $\tau_{\text {abs }}$. Then the sink term $S(r, t)=n / \tau_{a b s}$ and the Fokker-Planck equation is

$$
\frac{\partial \mathrm{n}}{\partial \mathrm{t}}=\mathrm{D} \frac{\partial^{2} \mathrm{n}}{\partial \mathrm{r}^{2}}-\frac{\mathrm{n}}{\tau_{\mathrm{abs}}}=0
$$

where, as above, $D$ is assumed to be constant throughout the ring and $\partial \mathrm{n} / \partial \mathrm{r}>>$ $2 n / r$. It is convenient to shift the origin by setting $r^{\prime}=r-r_{0}+D_{s^{\prime}}$, whereby $r^{\prime}=0$ at the inner edge of the ring. Equation 23 is unchanged and its general solution is

$$
n\left(r^{\prime}\right)=A e^{r^{\prime} / a}+B e^{-r^{\prime} / a}
$$

where $a=\left(D \tau_{a b s}\right)^{1 / 2}$ is a characteristic diffusion length which depends both on the diffusion coefficient and the average absorption time. As boundary conditions, we let $n$ be arbitrary at $r^{\prime}=D_{s}$ (the outer edge of the ring) but set $n=0$ at $r^{\prime}=0$ (the inner edge). This is equivalent to placing a sink at the inner edge of the ring, which then prevents the diffusion of particles back into the region of absorption. For these boundary conditions $\mathrm{B}=-\mathrm{A}$ and

$$
n\left(r^{\prime}\right)=A\left(e^{r^{\prime} / a}-e^{-r^{\prime} / a}\right)=2 A \sinh \left(r^{\prime} / a\right)
$$


The radial flow of particles is given by

$$
J\left(r^{\prime}\right)=-D \frac{\partial n}{\partial r^{\prime}}=-\frac{A d}{a}\left(e^{r^{\prime} / a}+e^{-r^{\prime} / a}\right)
$$

where a negative value of $J$ represents a net inward flow of particles.

The fraction $F$ of particles that can diffuse in past a satellite without being absorbed is given by the ratio of the inward flow at the inner and outer edges:

$$
\begin{aligned}
F & =\frac{J\left(r^{\prime}=0\right)}{J\left(r^{\prime}=D_{s}\right)}=\frac{2}{e^{D_{s} / a}+e^{-D_{s} / a}} \\
& =1 / \cosh \left(D_{s} / a\right)=1 / \cosh \left(2 \sqrt{\tau_{\text {diff }} / \tau_{a b s}}\right)
\end{aligned}
$$

since $\tau_{\text {diff }} / \tau_{\text {abs }}=\left(D_{s} / 2 a\right)^{2}$. Thus, if the characteristic diffusion length a is short compared to a satellite diameter, or equivalently, if the absorption time $\tau_{a b s}$ is short compared to the diffusion time, only a small fraction of the particles will survive. This fraction is given in Table 3 for particles near each of the inner satellites.

It is clear from the values of $\tau_{a b s}, \tau_{d i f f}$, and $F$ in Table 3 and Figure 5 that Callisto will have essentially no effect on trapped particles. However, if our value of $\mathrm{D}$ is reasonably accurate, given the assumptions of our study, Ganymede will exert a relatively small effect, Europa will reduce the flux of inwarddiffusing particles by several orders of magnitude, and Io and Amalthea will act as complete barriers to diffusion. As indicated earlier, we expect that the diffusion analysis would be primarily applicable to trapped protons. A sketch of the expected proton fluxes at Jupiter, therefore, is shown in Figure 6. An 


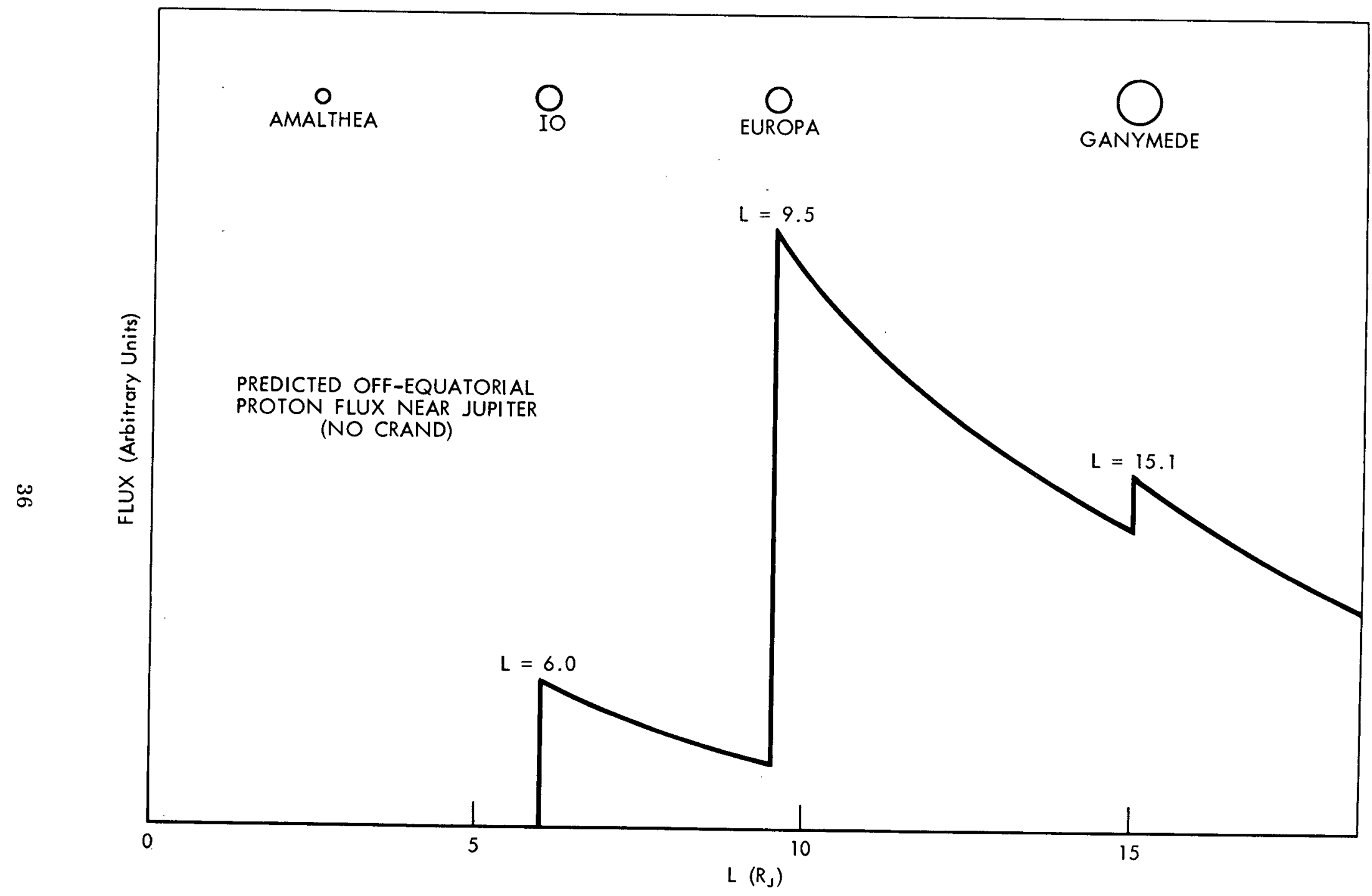

Figure 6. Sketch of expected off-equatorial proton fluxes at Jupiter, assuming diffusion is caused by magnetic fluctuations.

CRAND is ignored. Fluxes of inward-diffusing protons are expected to be reduced by several orders of magnitude at

Europa ( $L=9.5$ ), and none should diffuse past Io or Amalthea. 
implicit assumption, of course, is that all trapped protons diffuse in from the outer magnetosphere and that other sources, such as cosmic ray albedo neutron decay (CRAND), can be ignored.

We might ask whether particles can be moved radially past a satellite in one large jump by a single large sudden commencement. In their study of radial diffusion, Davis and Chang (1962) derived an equation for the intersection of a distorted line of force with the equatorial plane in a field given by Equation 13, where the solar wind moves the boundary into a distance $r_{b}$ :

$$
r=r_{0}\left(1-\frac{1}{2} \frac{a_{1}}{M}, \frac{r_{0}^{3}}{r_{b}^{3}}-\frac{8}{21} \frac{a_{2}}{M} \frac{r_{0}^{4}}{r_{b}^{4}} \cos \phi\right)
$$

where $r_{0}$ is the undistorted equatorial distance and $r_{0}<<r_{b}$. In Table 3 we show the value of $\Delta \mathbf{r}$ at noon $(\phi=0)$ obtained by moving the boundary from 50 to $40 \mathrm{R}_{\mathrm{J}}$ due to a sudden change in the solar wind. This would increase the contribution of the external field near Jupiter by a factor of about 2 . It is clear that particles adhering to a field line can easily move past Ganymede or Callisto. However, a sudden increase of this magnitude would move particles in by less than a satellite diameter at Europa, and much less than this at Io or Amalthea. Thus these three satellites would still act as a major barrier to diffusion.

Electric field diffusion. Fälthammar (1965), Cornwall (1968), Birmingham (1969), and others have stressed the possible importance of time-varying electric potential fields in diffusing trapped particles in towards the Earth. Fälthammar (1968) showed that the diffusion coefficient is given by 


$$
D_{E}=\frac{L^{6}}{2 B_{0}^{2}} \sum_{n}\left(P_{n}(L, \nu)\right)_{\nu=1 / \tau_{d}}
$$

where $P_{n}$ is the power spectrum of the $n^{\text {th }}$ Fourier coefficient (in azimuth angle) of the fluctuating electric field, evaluated at the drift frequency. To the extent that this power spectrum is independent of $\mathrm{L}$, the diffusion coefficient is proportional to $L^{6}$, instead of the $L^{10}$ dependence characteristic of magnetic fluctuations.

Since it is extremely difficult, even at Earth, to estimate the magnitude of the electric field diffusion coefficient, we will make no attempt to do so at Jupiter. However, in order to evaluate the effect of a possible $\mathrm{L}^{6}$ dependence, we have listed in Table 3 and plotted in Figure 5 the characteristic diffusion times near each of the inner satellites for a diffusion coefficient arbitrarily normalized to the same value as our estimated magnetic diffusion coefficient at $\mathrm{L}=10$, i.e.,

$$
D_{E}=3.4 \times 10^{-11} L^{6} R_{J}^{2} / \text { day }
$$

Such a coefficient would, of course, produce slower diffusion at $\mathrm{L}>10$ but more rapid diffusion at $\mathrm{L}<10$. For this assumed value, diffusion times near Amalthea, Io, and Europa are still much longer than the calculated absorption times. 


\section{EFFECT OF THE DIPOLE TILT}

The calculations above of the sweeping effect of Jupiter's satellites assumed that the magnetic dipole was aligned with the rotational axis. In this section we consider the effect of the 10-degree tilt of the dipole and calculate the resulting increased absorption times for near-equatorial particles. Particles which mirror at latitudes less than $10^{\circ}$ (equatorial pitch angles greater than $69^{\circ}$ ), if located at the proper longitude, could not impact a satellite. In the co-rotating jovimagnetic frame of reference shown in Figure 3 , a particle located at a jovimagnetic longitude of $0^{\circ}$ or $180^{\circ}$ (with the jovimagnetic prime meridian passing through the jovigraphic and jovimagnetic poles) would see the satellites passing at $10^{\circ}$ above or below the magnetic equator. However, protons would drift east and electrons west (Jupiter's magnetic field is opposite in direction to the Earth's) with the drift periods given in Table 2. As they drift, the satellites appear to pass by at lower latitudes, thus interacting with some of the particles with equatorial pitch angles greater than $69^{\circ}$. At the nodes $\left(\phi_{\mathrm{m}}=90^{\circ}\right.$ or $270^{\circ}$ ), even 90 -degree pitch-angle particles can impact the satellites. The average absorption lifetime is thus a function of pitch angle.

We first calculate the fraction of a drift period over which a particle with a given pitch angle can interact with a satellite. From Figure 3, the apparent magnetic latitude $\lambda_{\mathbf{s}}$ at which a satellite appears to pass by can be expressed in terms of the magnetic longitude of the particle $\phi_{\mathrm{m}}$ :

$$
\lambda_{\mathrm{s}} \approx 10^{\circ} \cos \phi_{\mathrm{m}}
$$


The relationship in a dipole field between mirror latitude $\lambda_{\mathrm{m}}$ and equatorial pitch angle $a_{e}$ is given by

$$
\sin ^{2} \alpha_{e}=\cos ^{6} \lambda_{m} /\left(1+3 \sin ^{2} \lambda_{m}\right)^{1 / 2}
$$

(Hess, 1968, Eq. 2.16). For near-equatorial particles this reduces to

$$
a_{\mathrm{e}} \approx 90 \pm(9 / 2)^{1 / 2} \lambda_{\mathrm{m}}=90 \pm 2.1 \lambda_{\mathrm{m}}
$$

Particles can interact with a satellite only if their mirror latitude $\lambda_{m}$ is greater than the satellite latitude $\lambda_{s}$, or, equivalently, considering pitch angles between $0^{\circ}$ and $90^{\circ}$ only,

$$
\left(90-\alpha_{e}\right) / 2.1>10^{\circ}\left|\cos \phi_{m}\right|
$$

Near the $90^{\circ}$ node, this condition is satisfied for longitudes such that

$$
\left|\phi_{\mathrm{m}}-90\right| \cdot<\sin ^{-1}\left(\frac{90-a_{\mathrm{e}}}{21}\right)
$$

and thus the fraction of a drift period, $g$, over which a particle can interact with a satellite is given by

$$
\begin{array}{ll}
g=\sin ^{-1}\left(\frac{90-a_{e}}{21}\right) / 90, & a_{e}>69^{\circ} \\
g=1, & a_{e} \leq 69^{\circ}
\end{array}
$$

This fraction depends only upon the equatorial pitch angle, and is given in Table 4 for particles of various pitch angles. (We do not consider here the case of pitch angles extremely close to $90^{\circ}$, where the finite diameter of the satellite places a lower limit on $\mathrm{g}$. 


\section{TABLE 4}

Effect of the 10-degree tilt on equatorial pitch-angle distributions. The parameter $\mathrm{g}$ is the fraction of a drift period in the co-rotating jovimagnetic coordinate system over which a particle can interact with a satellite; $\tau_{\text {abs }}^{\prime}$ is the modified absorption lifetime near the satellite Europa, as given by Equation 40; and F is the fraction of particles which survive absorption in diffusing past Europa, assuming a characteristic diffusion time, based on magnetic fluctuations, of 24 days. No pitch-angle scattering is assumed.

\begin{tabular}{cccccc}
\hline $\begin{array}{c}\text { Pitch } \\
\text { Angle }\end{array}$ & $\mathrm{g}$ & \multicolumn{3}{c}{ Protons } & \multicolumn{2}{c}{ Electrons } \\
\hline $69^{\circ}$ & 1.00 & $1.9 \mathrm{~d}$ & 0.0016 & $0.47 \mathrm{~d}$ & $1.2 \mathrm{E}-6$ \\
$70^{\circ}$ & 0.80 & 3.5 & 0.011 & 2.5 & 0.0043 \\
$75^{\circ}$ & 0.51 & 6.4 & 0.042 & 5.8 & 0.034 \\
$80^{\circ}$ & 0.32 & 9.7 & 0.086 & 8.3 & 0.066 \\
$85^{\circ}$ & 0.15 & 17.0 & 0.185 & 11.5 & 0.111 \\
$87^{\circ}$ & 0.09 & 25.8 & 0.284 & 14.2 & 0.147 \\
$89^{\circ}$ & 0.03 & 67.9 & 0.558 & 25.1 & 0.278 \\
\hline
\end{tabular}


Now from Tables 2 and 3 it is clear that the absorption lifetimes for protons and electrons are much less than the drift periods in the co-rotating system (i.e., $\tau_{\text {abs }}<<\tau_{d}$ ), except for protons near Amalthea. An approximate value for the modified absorption lifetime $\tau_{\text {abs }}^{\prime}$ can be given in each of three limiting cases:

Case $A: a_{\mathrm{e}} \leqslant 69^{\circ}, \mathrm{g} \approx 1$. In this case the analysis in the earlier section is applicable, and

$$
\tau_{\text {abs }}^{\prime}=\tau_{\text {abs }} \text { Case A }
$$

Case B: $69^{\circ}<a_{\mathrm{e}}<90^{\circ}$, but $\mathrm{g} \tau_{\mathrm{d}}>>\tau_{\mathrm{abs}}$. In this case the maximum time that a particle would be unable to impact a satellite, assuming it were injected at just the right longitude, would be $(1-\mathrm{g}) \dot{\tau}_{\mathrm{d}} / 2$ : On the average, however, a particle would survive about half this long (assuming, of course, that $\tau_{a b s}<<\tau_{d}$ ), and therefore

$$
\tau_{a b s}^{\prime}=(1-g) \tau_{d} / 4 \text { Case B }
$$

Case C: $\alpha_{\mathrm{e}} \approx 90^{\circ}, \mathrm{g} \tau_{\mathrm{d}}<<\tau_{\mathrm{abs}}$. In this case the particles have a good chance of drifting past the nodes without impacting the satellite, and the modified absorption lifetime is given by

$$
\tau_{a b s}^{\prime}=\tau_{a b s} / g \text { Case } \mathrm{C}
$$

A semi-empirical expression for $\tau_{\text {abs }}^{\prime}$ that gives nearly the correct result for each of these cases, yet makes a smooth transition between them, is

$$
\tau_{a b s}^{\prime}=(1-g) \tau_{d} / 4+\tau_{a b s} / g \text { A11 Cases }
$$

This expression is probably reasonably accurate, within a factor of two or three, for all values of $\mathrm{g}$. 
In Table 4 the values of $\tau_{\text {abs }}^{\prime}$ and F (as defined in an earlier section, based on magnetic diffusion) are given for particles of various pitch angles near the satellite Europa. The parameter $\mathrm{F}$ is plotted in Figure 7. The extremely sharp increase in this parameter at $a_{e}=69^{\circ}$ is due to the fact that absorption times suddenly increase from those given by Equation 37 (Case A) to times which are of the order of a fraction of a drift period $\tau_{d}$ (Case B).

The net effect would be a large change in the equatorial pitch-angle distributions on either side of the orbit of Europa. On the inner side the particles would have distributions sharply peaked near $90^{\circ}$. A spacecraft which passed by the planet at magnetic latitudes greater than $10^{\circ}$ would never see most of these particles. An important parameter in the analysis of the energetic particle measurements from Pioneer 10, which will fly by Jupiter in December, 1973, will be the jovimagnetic latitude $\lambda_{\mathrm{jm}}$ of the spacecraft. If $\lambda_{\mathrm{jm}}>10^{\circ}$ as it moves in past $\mathrm{L}=9.5$, and if the processes we assume here are dominant, we would predict a large sudden decrease in the measured omnidirectional flux. If $\lambda_{j \mathrm{~m}}$ is near $0^{\circ}$, we would expect reduced fluxes and/or strongly-peaked equatorial pitch-angle distributions at $\mathrm{L}<9.5$.

At Io and Amalthea, however, even though absorption lifetimes for particles with pitch angles greater than $69^{\circ}$ would be substantially increased, these lifetimes are still very much shorter than the characteristic diffusion times given in Table 3. Thus, if the basic assumptions of this study are correct, and if the calculated diffusion times are reasonably accurate, even near-equatorial particles cannot diffuse past Io or Amalthea.

However, we know from the analysis of the decimeter radiation that electrons with sharply-peaked pitch-angle distributions are present near $L=2$ (Thorne, 1965), 


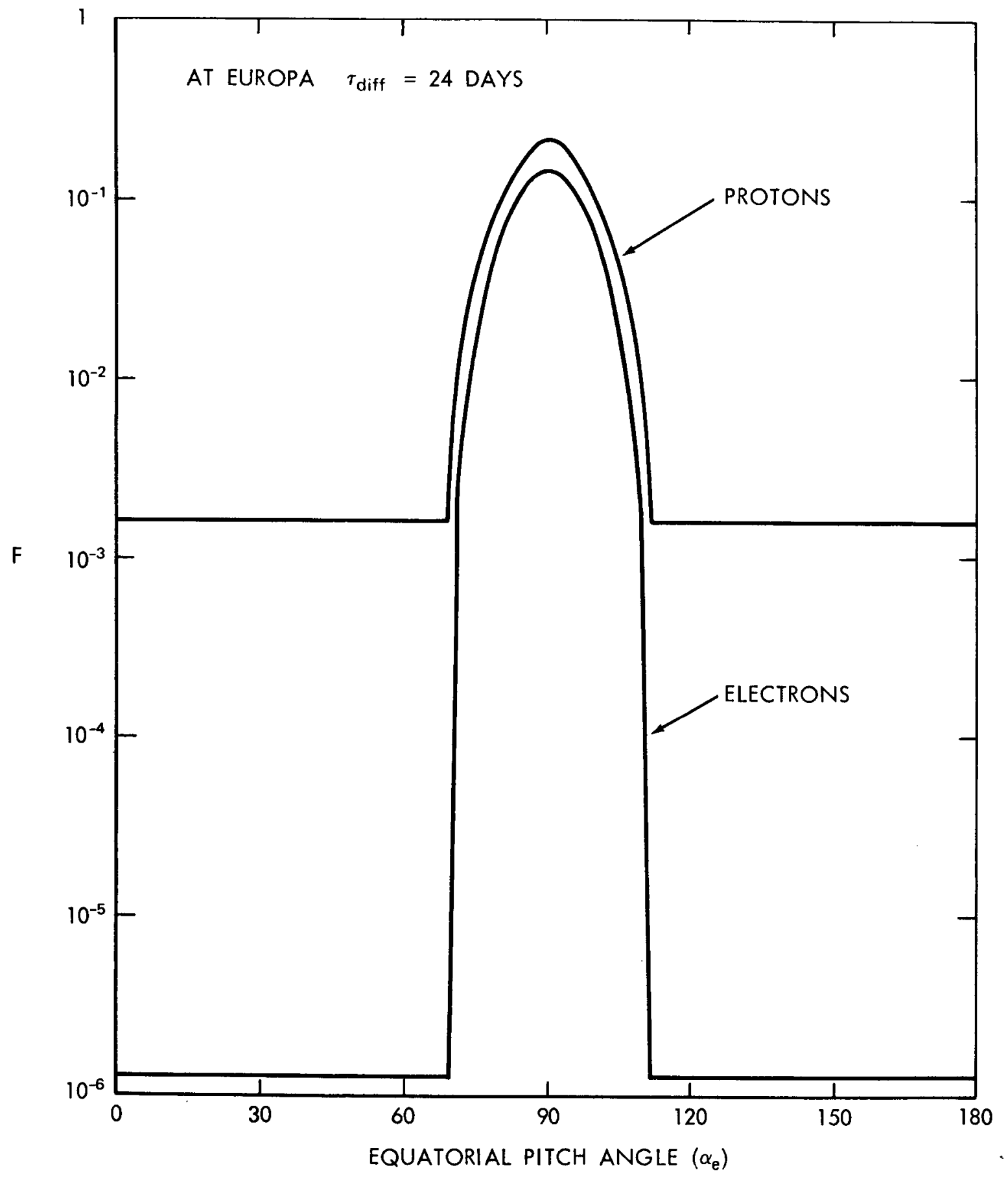

Figure 7. The fraction $E$ of particles that can diffuse in past Europa without being absorbed, plotted as a function of pitch angle. The characteristic diffusion lifetime is assumed to be 24 days. The increase at $69^{\circ}<a_{0}<111^{\circ}$ is due to the fact that during a substantial fraction of these particles'drift period, the satellites appear to pass by at latitudes above or below their mirror points. 
just inside the orbit of Amalthea. If there exists some kind of mechanism which diffuses electrons much more rapidly than we have calculated, those with pitch angles near $90^{\circ}$ would have a greater probability of surviving than those with pitch angles less than $69^{\circ}$, since the drift period for $50 \mathrm{Mev} /$ gauss electrons near Amalthea is about 17 days, compared with an absorption lifetime (Case 2) of 2.4 days. This effect could easily produce the kind of pitch-angle distributions required by Thorne. 


\section{DISCUSSION}

A number of additional simplifying assumptions were made in deriving the results of the earlier sections. Here we examine the consequences of removing some of these simplifications.

The existence of quadrupole and higher-order terms or a possible offset of the equivalent dipole would not affect our analysis in any significant way. The effect of a dipole offset would be to make the circular satellite orbits appear to be offset by an equivalent amount in the co-rotating jovimagnetic frame of reference shown in Figure 3. However, each satellite would come back to the same apparent position after one co-rotation period. For random absorption (Case 1), the same particles would be subject to absorption again (assuming no diffusion or longitudinal drift), and for snowplow absorption (Case 2), exactly the same path would be plowed out during each apparent orbit. A similar argument applies if we consider the presence of quadrupole or higher-order terms.

We can also argue that the slight eccentricity in the orbit of each satellite is unimportant. From the eccentricities listed in Table 1 we calculate that the maximum deviations from a circular orbit are $540,0,200$, and $1600 \mathrm{~km}$, respectively, for satellites Amalthea through Ganymede. For all but Amalthea these are much less than a satellite diameter.

The alternate compression and extension of a co-rotating field line by the solar wind during one jovian day is another possible source of radial motion of particles. Equation 28, giving the intersection of field lines with the magnetic equator, can be used to calculate the maximum diurnal deviations at noon $(\phi=0)$ 
and midnight $\left(\phi=180^{\circ}\right)$ from their average positions. These distances are 0.3 , 22, 220, and $2280 \mathrm{~km}$, for satellites Amalthea through Ganymede, respectively, assuming $r_{b}=50 R_{J}$. Again, these distances are much less than the respective satellite diameters, and can be ignored.

We have assumed that all trapped particles co-rotate with the planet. If at some radial distance this assumption does not hold, the apparent period of revolution of each satellite would be the sidereal period given in Table 1, rather than the co-rotation period. This would mean that the absorption of $50 \mathrm{Mev} /$ gauss protons would be governed by Case 2 instead of Case 1 at Europa and Ganymede, and the absorption times for both electrons and protons at Io, Europa, and Ganymede would increase to $1.8,3.6$, and 7.2 days, respectively, instead of the values given in Table 3. This relatively small increase would not have a significant effect on our findings.

We have assumed in this study that the source of Jupiter's radiation belts are solar wind particles which have diffused in from the outer magnetosphere. We have ignored possible additional source mechanisms, such as CRAND (cosmic ray albedo neutron decay). The flux of galactic cosmic rays incident on Jupiter's atmosphere is reduced by a factor of approximately 4000 from the corresponding flux at Earth, since the cosmic-ray cutoff ridigity at Jupiter is 360 times as large. Only cosmic-ray particles with energies greater than about $5 \mathrm{Tev}\left(5 \times 10^{12} \mathrm{ev}\right)$ can reach the surface of Jupiter at low latitudes. Thus the CRAND source strength will be several orders of magnitude less than at Earth. The loss rates will also be much lower, due to the lower density of thermal plasma and essentially complete absence of neutral atmosphere above a thousand 
kilometers. The resulting steady-state flux of trapped particles from CRAND will probably be no greater than, and probably less than, the flux of CRANDproduced particles at Earth. As with inward-diffusing particles, Amalthea at $\mathrm{L}=2.5$ and Io at $\mathrm{L}=6$ will act as barriers to the outward diffusion of these particles, unless our calculated diffusion times are substantially in error. 


\section{SUMMARY AND CONCLUSIONS}

In this study we have examined the competing processes of (1) absorption of trapped particles by impact with one of Jupiter's satellites and (2) diffusion of these particles by violation of the third adiabatic invariant. We have assumed that a particle would be removed from the radiation belt if, in its normal motion, it would happen to impact a satellite. We have assumed that the satellites are unmagnetized and that they would not interfere with the motion of a nearby particle in any way. With the additional assumption that Jupiter's dipole axis is aligned with its rotational axis, the average absorption lifetime of a proton or electron with magnetic moment $\dot{\mu}=50 \mathrm{Mev} /$ gauss was found to be $0.5-5$ days near Amalthea, Io, Europa, or Ganymede, and somewhat longer at Callisto (see Table 3).

A diffusion coefficient was then calculated, assuming violation of the third invariant due to magnetic fluctuations associated with fluctuations in the solar wind. We assumed that on the average there was one disturbance per (Earth) day which doubled the contribution of the external field near Jupiter. This led to a diffusion coefficient $\mathrm{D}$ equal to $0.13 \mathrm{r}^{10} / \mathrm{r}_{\mathrm{b}}^{8} \mathrm{day}^{-1}=3.4 \times 10^{-15} \mathrm{~L}^{10} \mathrm{R}_{\mathrm{J}}^{2} /$ day, taking the subsolar boundary distance $r_{b}=50 R_{J}$. A similar calculation for the Earth's magnetosphere, taking $r_{b}=10 R_{E}$, gives $D=1.3 \times 10^{-9} \mathrm{~L}^{10} \mathrm{R}_{E}^{2} /$ day, about equal to the value quoted by Williams (1972) as that value which could explain the behavior of steady-state and non-steady-state outer-belt protons at Earth.

A characteristic diffusion time in the vicinity of each satellite, equal to the mean time for a particle to diffuse by one satellite diameter, was computed. 
This ranged from 137 years at Amalthea to 0.002 days at Callisto (see Table 3 and Figure 5). This time was short compared to an average lifetime at Callisto, roughly equal to the absorption time at Ganymede, but substantially greater than the absorption time at Europa, Io, and Amalthea. The fraction of particles which survive absorption in diffusing past each satellite was computed (see Table 3). Amalthea and Io were found to act as complete barriers to diffusion, and Europa would be expected to reduce particle fluxes by three orders of magnitude or more. A small reduction would be observed at Ganymede, and Callisto would have essentially no effect. Figure 6 illustrates the predicted proton fluxes at Jupiter, showing the expected decreases at $\mathrm{L}=6$ and $\mathrm{L}=9.5$. Similar calculations were made for a diffusion coefficient proportional to $\mathrm{L}^{6}$ which might be produced by fluctuating electric fields, arbitrarily normalized to the value of the magnetic diffusion coefficient at $L=10$. The conclusions were essentially unchanged.

We then examined the effect of the 10-degree tilt of Jupiter's magnetic dipole axis. The absorption times were found to depend strongly upon equatorial pitch angle. For particles with pitch angles greater than $69^{\circ}$, mirroring at latitudes less than $10^{\circ}$, the average absorption times are increased from those calculated earlier to times which are a fraction of a drift period (see Table 4), since at some jovimagnetic longitudes near-equatorial particles cannot interact with any satellite. Figure 7 shows the fraction of particles that would survive absorption in diffusing past Europa as a function of pitch angle, assuming a characteristic diffusion time at Europa of 24 days. The equatorial pitch-angle distributions of particles just inside this satellite would thus be expected to be highly peaked near $a_{e}=90^{\circ}$. 
However, even for near-equatorial particles, the absorption lifetimes near Io and Amalthea are substantially shorter than diffusion times based on magnetic fluctuations produced by solar wind variations. Thus, given the assumptions of this study, solar wind particles diffusing inwards from the outer magnetosphere are not expected to be able to penetrate the inner satellites of Jupiter, particularly Amalthea and Io. Yet high fluxes of trapped electrons with energies of the order of $10 \mathrm{Mev}$ are definitely responsible for the synchrotron emission near $\mathrm{L}=2$. Are these solar-wind electrons, and if so, how did they get there? There are several possible explanations:

1. A satellite might in some way perturb the motion of a nearby electron, so that it would not impact the satellite. This could be due to an inherent magnetic field possessed by the satellite, or to the interaction of a highly-conducting satellite with the field lineswhich co-rotate past it. The strong effect which Io is observed to exert on the decametric radiation bursts indicate that it interacts strongly with the field lines at $\mathrm{L}=6$, giving some support to the latter hypothesis. Protons with the same magnetic moment as electrons will have a relativistic momentum everywhere 43 times greater (the square root of the mass ratio), and therefore the motion of protons is not likely to be affected nearly so much by local perturbations in the magnetic or electric fields. It is possible, therefore, that electrons could slip past the inner satellites but protons would be absorbed.

2. Diffusion of electrons in the inner magnetosphere might be much more rapid than the calculations based on magnetic fluctuations have indicated. If Amalthea and Io do not perturb the motion of nearby electrons, diffusion rates in this region would have to be increased by about four orders of magnitude for 
electrons to diffuse past these satellites without being absorbed. Additional processes, unknown at Earth, might be responsible for this rapid diffusion at Jupiter. Brice (1972) and Brice and McDonough (1972) have suggested that neutral atmospheric and ionospheric winds and turbulence at the top of Jupiter's atmosphere might provide the required driving mechanism.

If indeed the diffusion of electrons proceeds much more rapidly than indicated in this study near Io and Amalthea, the effect of the 10-degree tilt of the dipole will be for these satellites to preferentially remove particles with mirror latitudes greater than 10 degrees. Those that survive will have pitch-angle distributions strongly peaked near $90^{\circ}$; we believe that this is the most probable mechanism producing the sharply-peaked distributions required in the studies of Thorne (1965) and Clarke (1970), particularly since Amalthea at $\mathrm{L}=2.5$ is located just outside the peak of the synchrotron emission region. Without more precise knowledge of the processes causing enhanced diffusion, however, we cannot say whether protons would be affected by the same rapid diffusion.

3. Additional unknown acceleration processes might give rise to energetic electrons in the inner magnetosphere. We know that energetic electrons are sometimes injected at low L-values at Earth following a major magnetic storm (e.g., Williams et al., 1968). Similar processes might operate at Jupiter. It is not as likely, however, that energetic trapped protons could be produced by such means.

We probably will not be able to determine which alternative best explains the existence of energetic electrons at $L=2$, or whether substantial fluxes of protons are able to diffuse past the satellites, until actual particle measurements 
are made at Jupiter by Pioneer 10 and later spacecraft. It will be very important for the onboard detectors to distinguish clearly between trapped electrons and protons, as there is good reason to believe that they might behave very differently. It will be particularly important to get accurate flux measurements near $\mathrm{L}=5.95$, 9.47, and 15.1, the positions of Io, Europa, and Ganymede. Accurately-measured pitch-angle distributions, particularly if the flyby is at low magnetic latitudes, will be extremely useful in evaluating the various hypotheses.

Finally, if a Jupiter flyby is planned into the very inner regions of its magnetosphere, and if for the safety of the spacecraft the lowest possible radiation levels are desired, the mission should be planned so that the spacecraft remains at relatively high magnetic latitudes, so as to avoid intense fluxes of particles with equatorial pitch angles near $90^{\circ}$. 


\section{Acknowledgments}

We wish to thank Theodore Northrop and Thomas Birmingham for reading and commenting critically on the manuscript, and James Warwick for many helpful discussions. We also thank Andrew Beck for the opportunity to present the initial development of these ideas before a critical audience at the JPL Jupiter Radiation Belt Workshop in 1971. 


\section{REFERENCES}

Beck, A. J., Jupiter trapped radiation belts, JPL Quarterly Technical Review, $\underline{1}, 78-88,1972$.

Berge, G. L., An interferometric study of Jupiter's decimeter radio emission, Astrophys. J., 146, 767-

Berge, Glenn L., Some recent observations and interpretations of the Jupiter decimeter radiation, in Proceedings of the Jupiter Radiation Belt Workshop, edited by A. J. Beck, pp. 223-242, Jet Propulsion Laboratory Technical Memorandum 33-543, July, 1972.

Birmingham, Thomas J., Convection electric fields and the diffusion of trapped magnetospheric radiation, J. Geophys. Res., 74, 2169-2181, 1969.

Birmingham, T. J., T. G. Northrop, and C. G. Fälthammar, Charged particle diffusion by violation of the third adiabatic invariant, Phys. Fluids, 10, 2389-2398, 1967.

Blanco, V. M., and S. W. McCuskey, Basic Physics of the Solar System, AddisonWesley Pub. Co., 1961.

Bransom, N. J. B. A., High resolution radio observations of the planet Jupiter, Mon. Not. R. Astr. Soc., 139, 155-162, 1968.

Brice, Neil M., Bulk motion of the magnetosphere, J. Geophys. Res., $\underline{72}$, 5193$5211,1967$.

Brice, Neil M., and George A. Ioannidis, The magnetospheres of Jupiter and Earth, Icarus, 13, 173-183, 1970. 
Brice, Neil M., Energetic protons in Jupiter's radiation belts, in Proc. Jupiter Radiation Belt Workshop, edited by A. J. Beck, pp. 283-314, Jet Propulsion Laboratory Technical Memo 33-543, July, 1972.

Brice, N., and T. McDonough, Jovian ionospheric winds and radiation belt, Trans. Amer. Geophys. Union, 53, 485, 1972.

Carr, Thomas D., and Samuel Gulkis, The magnetosphere of Jupiter, Ann. Rev. Astr. and Astrophys., 7, 577-618, 1969.

Chang, David B., and Leverett Davis, Jr., Synchrotron radiation as the source of Jupiter's polarized decimeter radiation, Astrophys. J., 136, 567-581, 1962 .

Clarke, J. N., A synchrotron model for the decimetric radiation of Jupiter, Radio Sci., 5, 529-533, 1970.

Cornwall, J. M., Diffusion processes influenced by conjugate-point wave phenomena, Radio Sci., 3, 740-744, 1968.

Cornwall, John M., Radial diffusion of ionized helium and protons: a probe for magnetospheric dynamics, J. Geophys. Res., 77, 1756-1770, 1972.

Davis, Leverett, Jr., and D. B. Chang, On the effect of geomagnetic fluctuations on trapped particles, J. Geophys. Res., 67, 2169-2179, 1962.

Davis, Leo R., and J.M. Williamson, Low-energy trapped protons, Space Res., $\underline{3}, 365-375,1963$.

Divine, Neil, The Planet Jupiter (1970), NASA Space Vehicle Design Criteria (Environment), NASA SP-8069, December, 1971. 
Divine, Neil, Jupiter radiation belt engineering model, in Proc. National Symposium on Natural and Manmade Radiation in Space, NASA TM X-2440, 1972.

Dollfus, A., New optical measurements of the diameters of Jupiter, Saturn, Uranus, and Neptune, Icarus, 12, 101-117, 1970a.

Dollfus, A., Diamètres des planètes et satellites, in Surfaces and Interiors of Planets and Satellites, Ed. by A. Dollfus, pp. 46-139, Academic Press, 1970 b.

Donivan, F. F., and T. D. Carr, Jupiter's decametric rotation period, Astrophys. J., 157, L65-L68, 1969.

Fälthammar, Carl-Gunne, Effects of time-dependent electric fields on geomagnetically trapped radiation, J. Geophys. Res., 70, 2503-2516, 1965.

Fälthammar, Carl-Gunne, On the transport of trapped particles in the outer magnetosphere, J. Geophys. Res., 71, 1487-1491, 1966.

Fälthammar, Carl-Gunne, Radial diffusion by violation of the third adiabatic invariant, in Earth's Particles and Fields, edited by B. M. McCormac, pp. 157-169, Reinhold Publishing Co., New York, 1968.

Farley, Thomas A., and Martin Walt, Sources and loss processes of protons in the inner radiation belt, J. Geophys. Res., 76, 8223-8240, 1971.

Field, George B., The source of radiation from Jupiter at decimeter wavelengths, J. Geophys. Res.; 64, 1169-1177, 1959.

Hess, Wilmot N., The Radiation Belt and Magnetosphere, Blaisdell Püb. Co., 1968.

Luthey, Joe L., and David B. Beard, The electron energy and density distribution in the Jovian magnetosphere, Astrophys. J., to be published, 1972. 
Mead, Gilbert D., Deformation of the geomagnetic field by the solar wind, J. Geophys. Res., 69, 1181-1195, 1964.

Mead, Gilbert D., The effect of Jupiter's satellites on the diffusion of protons, in Proc. Jupiter Radiation Belt Workshop, edited by A. J. Beck, pp. 271-282, Jet Propulsion Laboratory Technical Memorandum 33-543, July, 1972.

Nakada, M. P., J. W. Dungey, and W. N. Hess, On the origin $6 f$ outer-belt protons, J. Geophys. Res., 70, 3529-3532, 1965.

Nakada, M. P., and G. D. Mead, Diffusion of protons in the outer radiation belt, J. Geophys. Res., 70, 4777-4791, 1965.

Newkirk, L. L., and M. Walt, Radial diffusion coefficient for electrons at 1.76 $<\mathrm{L}<5$, J. Geophys. Res., 73, 7231-7236, 1968.

Roberts, J. A., and M. M. Komesaroff, Observations of Jupiter's radio spectrum and polarization in the range from $6 \mathrm{~cm}$ to $100 \mathrm{~cm}$, Icarus, 4, 127-156, 1965.

Singer, S. F., Radiation belts of Venus and of Mars (with consideration of sweeping effect of Phobos), in Space Age Astronomy, edited by A. J. Deutsch and W. B. Klemperer, pp. 444-461, Academic Press, 1962.

Sloanaker, R. M., Apparent temperature of Jupiter at a wavelength of $10 \mathrm{~cm}$, Astron. J., 64, 346, 1959.

Thorne, Kip S., Dependence of Jupiter's decimeter radiation on the electron distribution in its Van Allen belts, Radio Science, 69D, 1557-1560, 1965.

Tverskoy, B. A., Transport and acceleration of charged particles in the Earth's magnetosphere, Geomagnetism and Aeronomy, 5, 617-628, 1965. 
Walt, Martin, Radial diffusion of trapped particles and some of its consequences, Rev. Geophys. Space Phys., 9, 11-25, 1971a.

Walt, Martin, The radial diffusion of trapped particles induced by fluctuating magnetospheric fields, Space Sci. Rev., $\underline{12}, 446-485,1971 \mathrm{~b}$.

Warwick, James W., Radio emission from Jupiter, Ann. Rev. Astron. Astrophys., $\underline{2}, 1-22,1964$.

Warwick, James W., Radiophysics of Jupiter, Space Sci. Rev., 6, 841-891, 1967.

Warwick, James W., Particles and Fields near Jupiter, NASA CR-1685, October, 1970 .

Williams, Donald J., Sources, losses, and transport of magnetospherically trapped particles, in Solar Terrestrial Physics/1970, Part III, edited by E. R. Dyer, pp. 66-130, D. Reidel Pub. Co., Dordrecht-Holland, 1972.

Williams, D. J., J. F. Arens, and L. J. Lanzerotti, Observations of trapped electrons at low and high altitudes, J. Geophys. Res., 73, 5673-5696, 1968. 